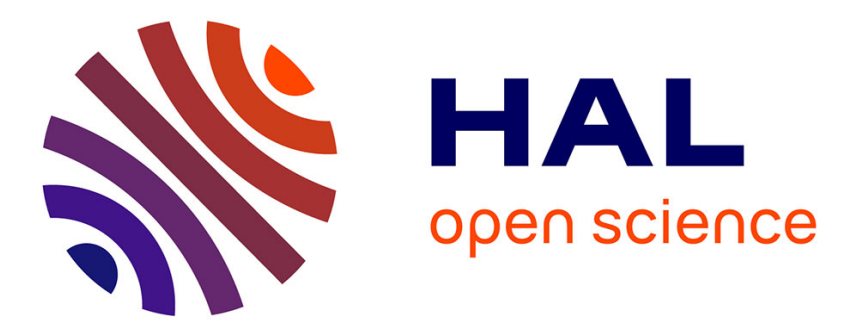

\title{
Non-linéarités optiques de suspensions de vésicules phospholipidiques unilamellaires analysées par une expérience de conjugaison de phase
}

\author{
E. Laffon, J.-P. Delville, W. Claeys, A. Ducasse
}

\section{- To cite this version:}

E. Laffon, J.-P. Delville, W. Claeys, A. Ducasse. Non-linéarités optiques de suspensions de vésicules phospholipidiques unilamellaires analysées par une expérience de conjugaison de phase. Journal de Physique II, 1992, 2 (5), pp.1073-1080. 10.1051/jp2:1992187 · jpa-00247693

\section{HAL Id: jpa-00247693 https://hal.science/jpa-00247693}

Submitted on 1 Jan 1992

HAL is a multi-disciplinary open access archive for the deposit and dissemination of scientific research documents, whether they are published or not. The documents may come from teaching and research institutions in France or abroad, or from public or private research centers.
L'archive ouverte pluridisciplinaire HAL, est destinée au dépôt et à la diffusion de documents scientifiques de niveau recherche, publiés ou non, émanant des établissements d'enseignement et de recherche français ou étrangers, des laboratoires publics ou privés. 
Classification

Physics Abstracts

$42.65 \mathrm{~F}-42.65 \mathrm{~J}$

\title{
Non-linéarités optiques de suspensions de vésicules phospholipidiques unilamellaires analysées par une expérience de conjugaison de phase
}

\author{
E. Laffon ( $\left.{ }^{1}\right)$, J.-P. Delville ( $\left.{ }^{(}\right)$, W. Claeys $\left({ }^{2}\right)$ et A. Ducasse ( $\left.{ }^{1}\right)$ \\ ( ${ }^{1}$ Centre de Physique Moléculaire Optique et Hertzienne, Université de Bordeaux I, \\ 33405 Talence Cedex, France \\ (2) I.X.L., Université de Bordeaux I, 33405 Talence Cedex, France
}

(Reçu le 23 juillet 1991, révisé le 21 novembre 1991, accepté le 30 janvier 1992)

\begin{abstract}
Résumé. - Pour la première fois à notre connaissance, nous avons mis en évidence des nonlinéarités optiques dans une suspension de vésicules phospholipidiques unilamellaires. Ces particules à intérêt biologique offrent la possibilité d'utiliser l'approximation de Born pour analyser les non-linéarités d'origine électrostrictive observées dans une expérience de conjugaison de phase (mélange dégénéré à quatre ondes), utilisant un laser continu à argon ionisé.
\end{abstract}

\begin{abstract}
For the first time to our knowledge, we report on optical nonlinearities of unilamellar phospholipid vesicle suspensions. These particles of biological interest allow the Born approximation to be used to analyse the nonlinearities of electrostrictive origin evidenced by a phase conjugation experiment (Degenerate-Four-Wave-Mixing), using a c.w. argon laser.
\end{abstract}

L'interaction laser-suspension colloïdale peut prendre plusieurs aspects suivant l'échelle à laquelle on choisit d'adapter les paramètres du faisceau lumineux gaussien issu d'un laser continu. Lorsque la dimension transversale du faisceau est très supérieure à la taille des particules en suspension, cette dernière se comporte comme un milieu caractérisé par un indice de réfraction non linéaire élevé, ainsi que l'avait prévu Palmer [1]. Lorsque cette dimension est de l'ordre de grandeur d'une particule, le laser permet de la piéger [2]. Cette dernière qualité de véritable pincette optique a fait l'objet d'applications spectaculaires dans le domaine biologique avec le piégeage de bactéries, de virus ou d'organites cellulaires [3], applications dont on commence à peine à exploiter l'extraordinaire richesse. Le travail présenté dans cet article concerne l'indice de réfraction non linéaire d'une suspension de particules à intérêt biologique dans le régime où leur taille est très inférieure au beam-waist du faisceau laser utilisé. Nous montrons qu'une suspension de vésicules phospholipidiques unilamellaires (VPU) présente de fortes non-linéarités optiques par une expérience de conjugaison de phase (mélange dégénéré à quatre ondes). Ces structures à intérêt biologique offrent ainsi également un intérêt pour l'optique non linéaire.

Une VPU peut être considérée comme un modèle simple de membrane cellulaire, formée uniquement de molécules phospholipidiques. A cause de leur caractère amphiphile, ces 
molécules s'organisent en milieu aqueux spontanément en bicouches. Une telle vésicule est représentée schématiquement en figure 1 . La préparation des suspensions a été réalisée avec un appareil de type EXTRUDER LIPEX 1,5 ml. Le passage d'une suspension aqueuse de phosphadidylcholine d'œuf (EPC en tampon Tris. $20 \mathrm{mM}$, EDTA $1 \mathrm{mM}$, pH 7,5) à travers une membrane de polycarbonate poreuse, sous une pression adaptée d'azote, conduit à une suspension de vésicules calibrées suivant la porosité de la membrane de polycarbonate choisie [4]. Dans nos expériences, nous avons préparé des suspensions de vésicules de $50 \mathrm{~nm}$ de rayon pour une épaisseur de la bicouche de $5 \mathrm{~nm}$, et dont les fractions volumiques se situaient entre $3 \%$ et $8 \%$ suivant le dosage initial des phospholipides $(5 \mathrm{mg}$ ou $10 \mathrm{mg}$ d'EPC par $\mathrm{ml}$ de tempon). Ces suspensions sont stables à l'échelle de la journée de travail.

Les VPU seront assimilées à de fines coquilles diélectriques sphériques dont l'indice de réfraction de la membrane dans son ensemble est évalué à 1,46 [5]. De telles VPU, quoique l'épaisseur de la membrane soit relativement petite par rapport à leur taille, possèdent néanmoins une polarisabilité positive non négligeable :

$$
\alpha=\frac{b^{3}\left(\varepsilon_{2}-\varepsilon_{1}\right)\left(2 \varepsilon_{2}+\varepsilon_{1}\right)\left[1-\left(\frac{a}{b}\right)^{3}\right]}{\left(2 \varepsilon_{2}+\varepsilon_{1}\right)\left(2 \varepsilon_{1}+\varepsilon_{2}\right)-2\left(\frac{a}{b}\right)^{3}\left(\varepsilon_{1}-\varepsilon_{2}\right)^{2}}
$$

valeur de la polarisabilité dans la limite Rayleigh, $b$ et $a$ représentant les rayons externe et interne de la coquille sphérique, $\varepsilon_{1}$ et $\varepsilon_{2}$ respectivement les constantes diélectriques du milieu continu et de la membrane.

Soit donc $\alpha=2,93 \times 10^{-24} \mathrm{~m}^{3}$ pour les dimensions des VPU décrites plus haut et en prenant l'indice de réfraction du milieu continu égal à 1,33 .

En conséquence, une suspension aqueuse de VPU pourra être considérée comme un gaz (parfait en première approximation), gaz responsable de non-linéarités d'origine électrostrictive décrites par Palmer [1]. Les particules, par couplage du champ électrique excitateur avec les moments dipolaires induits, et quoique toujours soumises à l'agitation thermique (à la

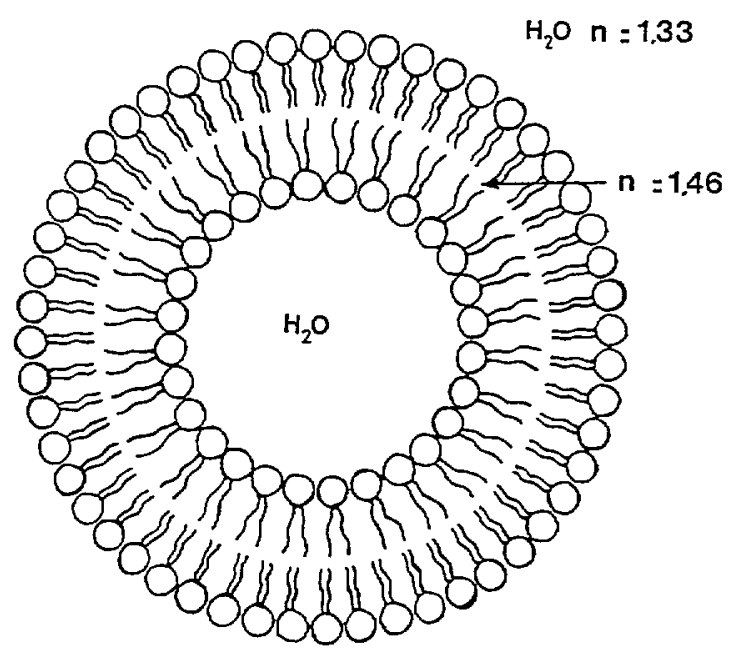

Fig. 1. - Coupe d'une vésicule phospholipidique unilamellaire.

[Sketch of a unilamellar phospholipid vesicle.] 
différence avec un piégeage individuel), se concentreront aux maxima de champ. Si $N$ est leur concentration en l'absence de champ, la variation $\mathrm{d} N$ de cette concentration dans un champ d'amplitude $E$, est telle que :

$$
\frac{\mathrm{d} N}{N} \simeq \frac{1}{2} \frac{\alpha E^{2}}{k T} \quad \text { si } \quad \frac{\mathrm{d} N}{N} \ll 1
$$

où $k$ est la constante de Boltzman, et $T$ la température du milieu.

L'indice de réfraction non linéaire $n_{2}$ est donné à vecteur d'onde q nul, c'est-à-dire pour une diffusion de la lumière strictement vers l'avant, par l'expression [1] :

$$
n_{2} \simeq \frac{\alpha^{2} N}{\varepsilon_{1} 2 k T c} \quad(\mathrm{MKSA})
$$

où $c$ représente la vitesse de la lumière dans le vide. Soit pour une préparation dont la fraction volumique est de $3 \%\left(N=0,5 \times 10^{14} \mathrm{VPU} / \mathrm{ml}\right)$, l'indice non linéaire attendu est : $n_{2} \approx 0,25 \times 10^{-11} \mathrm{~cm}^{2} / \mathrm{W}$.

Le montage expérimental est représenté en figure 2. Ce montage conduit à faire interférer les beam-waists de trois faisceaux $(125 \mu \mathrm{m})$ issus d'un laser $\mathrm{Ar}^{+}$(longueur d'onde $\lambda=514,5 \mathrm{~nm}$ ) au niveau de l'échantillon que l'on veut étudier [6]. Deux des faisceaux, dits pompe et d'intensité égale $\left(I_{\mathrm{p}}\right)$, se propagent exactement en sens inverse. Ils interfèrent avec le troisième, dit sonde $\left(I_{\mathrm{s}}\right)$, de moindre intensité que les deux premiers, et qui fait un angle $\Theta$ avec eux. L'intersection des faisceaux a lieu au niveau d'un capillaire rectangulaire en verre $\left(0,3 \times 4 \mathrm{~mm}^{2}\right)$, afin de limiter les effets convectifs dans la cellule de quartz Hellma (2 mm d'épaisseur) où ce capillaire est placé. Dans une expérience de conjugaison de phase par mélange dégénéré à quatre ondes l'onde conjuguée $\left(I_{c}\right)$ qui est engendrée au niveau de cette intersection a la propriété de suivre exactement le trajet inverse du faisceau sonde, trajet sur lequel une lame semitransparente permet de l'extraire. Ce faisceau est régulièrement ouvert et fermé à l'aide d'un système mécanique. Ce dispositif permet d'étudier la dynamique et la statique du signal conjugué. Un photomultiplicateur qui recueille le signal, est couplé à un analyseur multicanal qui assure son enregistrement. La sommation d'une centaine de ces enregistrements aboutit à l'obtention d'un signal moyenné exploitable, dont un exemple est donné en figure 3. La dynamique de ce signal montre, aussi bien pour sa constitution que pour sa relaxation, l'existence de deux constantes de temps très différentes.

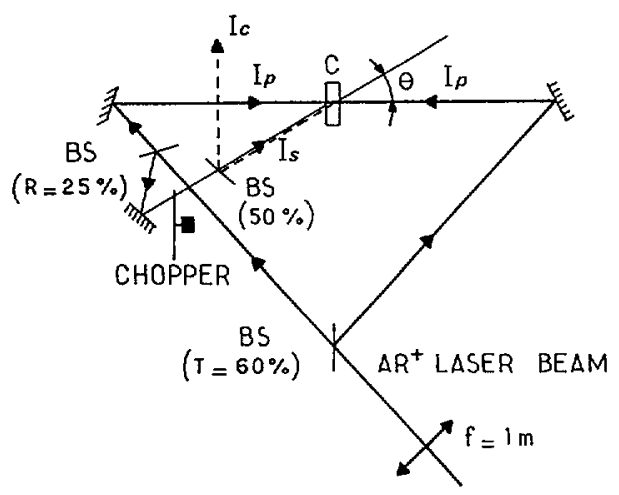

Fig. 2. - Représentation schématique du dispositif expérimental. BS : lame séparatrice; C : cellule.

[Schematic diagram of the experimental set-up. BS : beam splitter ; $\mathrm{C}:$ cell.] 


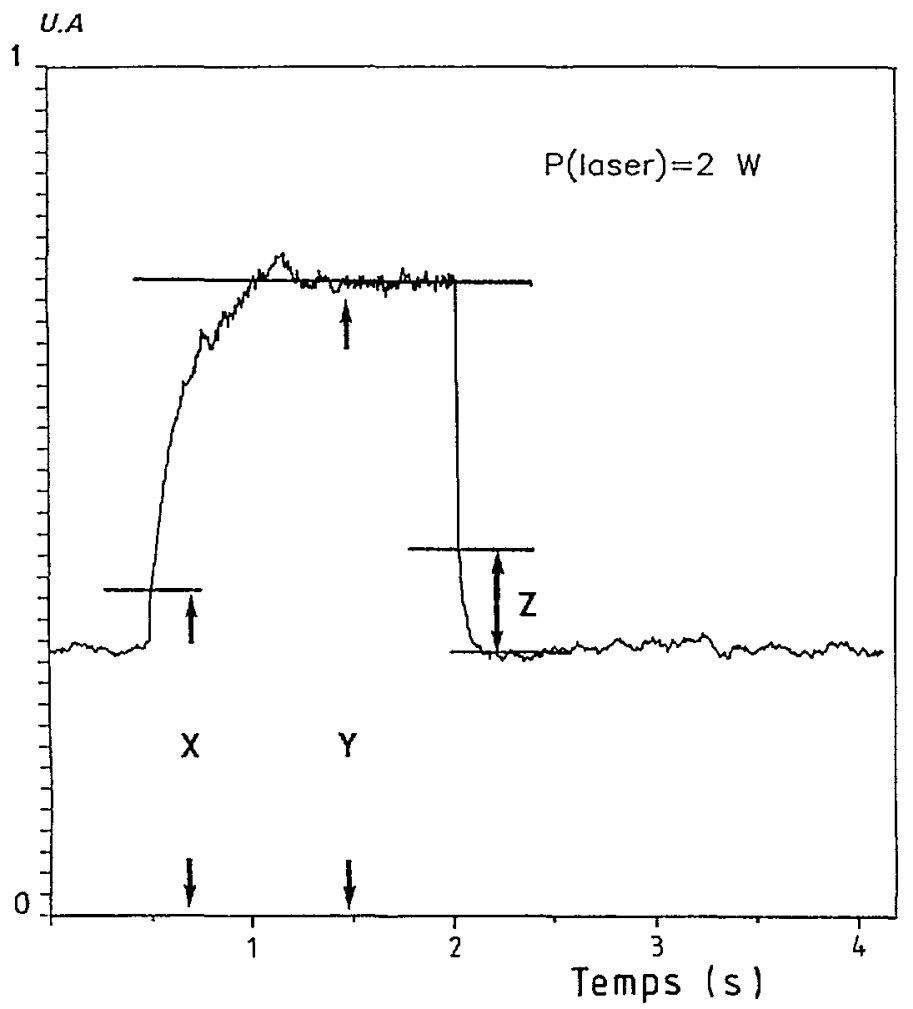

Fig. 3. - Enregistrement d'un signal conjugué typique $\left(\Theta=6,7^{\circ}\right)$. L'évaluation des trois paramètres $X, Y, Z$ permet de comparer les contributions des deux réseaux créés.

[Typical D.F.W.M. signal in a VPU suspension $\left(\Theta=6.7^{\circ}\right)$. The three parameters, $X, Y, Z$ allows us to compare the contributions of the two gratings.]

La génération d'une onde conjuguée dans le milieu colloüdal, se fait par la création de deux réseaux perpendiculaires d'interférences lumineuses entre le faisceau sonde et chaque faisceau pompe. A ces deux réseaux correspondent deux vecteurs d'onde d'excitation du milieu perpendiculaires $\mathbf{q}_{1}\left(q_{1}=\frac{2 \pi}{\Lambda_{1}}\right)$ et $\mathbf{q}_{2}\left(q_{2}=\frac{2 \pi}{\Lambda_{2}}\right)$ non nuls et de pas $\Lambda_{1}$ et $\Lambda_{2}$ très différents $\left(\Lambda_{1} \ll A_{2}\right)$, si l'angle $\Theta$ est petit. Sur ces deux réseaux se mettent en place (et relaxent à la coupure du faisceau sonde) deux réseaux de concentration en particules indépendants. L'onde conjuguée a donc deux contributions distinctes, correspondant aux réflexions dans la même direction et en condition de Bragg de chacun des faisceaux pompe sur le réseau de particules à la création duquel il n'a pas participé. Les temps caractéristiques des dynamiques respectives aux deux réseaux $\left(D q_{1}^{2}\right)^{-1} \ll\left(D q_{2}^{2}\right)^{-1}$ sont très différents puisque $\Lambda_{1} \ll \Lambda_{2}$, ce qui explique l'allure du signal de la figure 3 . $D$ est le coefficient de diffusion massique des particules $: D=\frac{k T}{(6 \pi \cdot r \cdot \eta)}$ où $r$ et $\eta$ sont le rayon des particules et la viscosité du solvant. Les variations de concentration en particules aux extrema de champ peuvent se constituer sous l'effet de deux processus : le processus électrostrictif déjà mentionné, mais aussi un processus thermodiffusif (effet Soret) comme l'ont prouvé différentes expériences menées avec des mélanges moléculaires [7] et avec une microémulsion critique [8]. Une expérience de conjugaison de phase sur un tel milieu [6] a confirmé l'intervention d'un effet 
Soret dans la constitution du réseau de grand pas uniquement. En effet, si d'une part l'absorption du milieu est suffisante et que d'autre part le pas du réseau est suffisamment large, alors se créent des gradients de température entre les maxima de champ. Cette contribution est non locale, car la température du milieu en un point dépend du profil du champ autour du point considéré. Elle se traduit quantitativement par une variation de la réflectivité du réseau en question qui est fonction du carré du pas de ce réseau [6]. Concernant notre milieu, l'existence d'un effet Soret est inconnue. Cependant, selon Lhuillier [9], la taille des VPU est un paramètre important qui indique que l'effet thermodiffusif est négligeable. Ainsi le processus électrostrictif local, car la variation de concentration en particules en un point ne dépend que de la valeur du champ en ce point, est-il le seul effet attendu.

En raison de la faible épaisseur de la bicouche phospholipidique, les modifications de la constante diélectrique du milieu associées à un réseau, $\Delta \in\left(\mathbf{q}_{1}, t\right)$, sont supposées faibles. L'approximation de Born permet alors d'exprimer le champ électrique diffusé, $E_{\mathrm{d}}(t)$, dans la direction caractérisée par le vecteur d'onde $\mathbf{q}_{1}$, de la façon suivante :

$$
E_{\mathrm{d}}(t) \propto E . \Delta \in\left(\mathrm{q}_{\mathrm{i}}, t\right)
$$

où $E$ est l'amplitude de l'onde pompe incidente sur le réseau considéré. $\Delta \in\left(\mathbf{q}_{1}, t\right)$ peut être explicité à l'aide de la fonction de forme associée aux vésicules :

$$
f\left(\mathbf{q}_{\mathrm{i}}\right) \propto \int_{V} \exp \left(i\left(\mathbf{q}_{\mathrm{i}} \cdot \mathbf{r}\right)\right) \cdot \mathrm{d} \mathbf{r}
$$

où $V$ est le volume de la particule présentant un contraste d'indice avec le solvant. Ainsi, si à $t=0$ on ouvre le faisceau sonde, et qu'à $t=T$ on l'obture après que le réseau ait atteint son état stationnaire, alors la dynamique de la contribution au signal conjugué de ce dernier au cours de ce cycle s'écrit à l'aide de $\Delta \in\left(\mathbf{q}_{\mathrm{i}}, t\right)$ :

$$
\begin{array}{rlrl}
0<t<T & \Delta \in\left(\mathbf{q}_{1}, t\right)=A \cdot \alpha^{2} & P \cdot\left|f\left(\mathbf{q}_{\mathrm{i}}\right)\right|^{2} & \left(1-\exp \left(-D q_{1}^{2} t\right)\right) \\
t>T & \Delta \in\left(\mathbf{q}_{1}, t\right)=A \cdot \alpha^{2} & P \cdot\left|f\left(\mathbf{q}_{\mathrm{i}}\right)\right|^{2} & \exp \left(-D q_{1}^{2}(t-T)\right)
\end{array}
$$

où $A$ est une constante et $P$ est la puissance à la sortie du laser.

Nous avons d'abord vérifié que l'évolution de l'intensité du signal conjugué était bien compatible avec une loi en puissance troisième de l'intensité du laser pour les valeurs utilisées dans nos enregistrements qui se situent entre 1,5 et $2 \mathrm{~W}$ à la sortie du laser (Fig. 4).

Nous avons alors étudié le rapport $R$, à l'état stationnaire, des modifications de la constante diélectrique relatives aux deux réseaux créés: soit $R=\Delta \varepsilon\left(\mathbf{q}_{2}, T\right) / \Delta \varepsilon\left(\mathbf{q}_{1}, T\right)=$ $\left|f\left(\mathbf{q}_{2}\right) / f\left(\mathbf{q}_{1}\right)\right|^{2}$ théoriquement. L'accès à ce rapport théorique est immédiat dès lors que l'on connaît l'expression du facteur de forme [10] :

$$
f(q) \propto \frac{1}{q^{3}\left(b^{3}-a^{3}\right)}[\sin q b-q b \cos q b-\sin q a+q a \cos q a] .
$$

Nous avons recherché expérimentalement ce rapport en faisant largement varier l'angle $\Theta$.

Pour dix valeurs de cet angle situées entre $9,5^{\circ}$ et $4,2^{\circ}, \Lambda_{1}$ reste pratiquement constant $\left(A_{1} \simeq \frac{\lambda}{2 \times 1,33} \simeq 0,19 \mu \mathrm{m}\right)$ alors que $A_{2}$ évolue de 3,1 à $7 \mu \mathrm{m}$. $R$ peut être évalué à partir d'un signal enregistré (voir Fig. 3) à l'aide des trois paramètres $X, Y, Z$, qui sont en relation avec les états stationnaires des réseaux :

$$
\begin{aligned}
X & =p\left[\Delta \varepsilon\left(\mathbf{q}_{1}, T\right)\right]^{2}+\text { Bruit } \\
Y & =p\left[\Delta \varepsilon\left(\mathbf{q}_{1}, T\right)+\Delta \varepsilon\left(\mathbf{q}_{2}, T\right)\right]^{2}+\text { Bruit } \\
Z & =p\left[\Delta \varepsilon\left(\mathbf{q}_{2}, T\right)\right]^{2}
\end{aligned}
$$




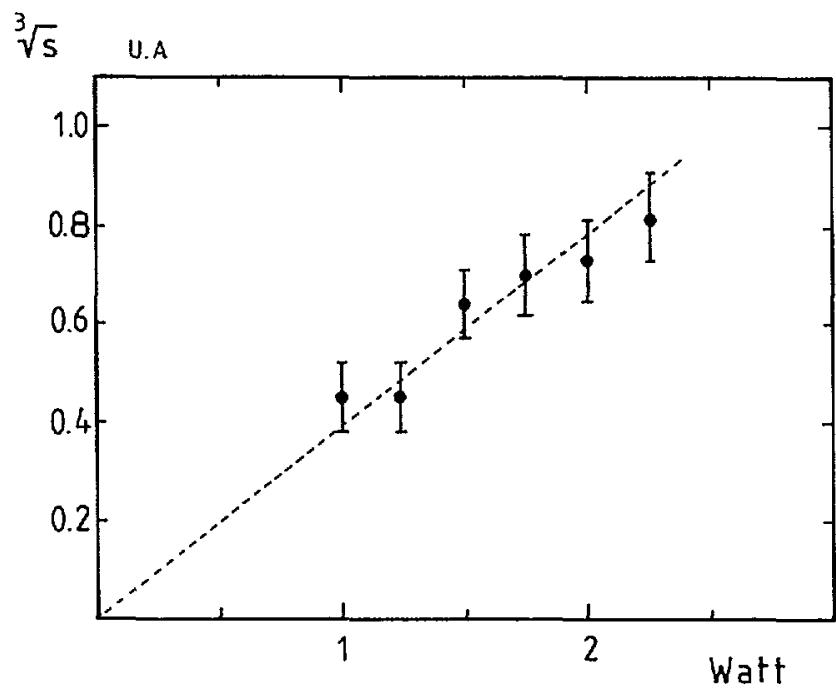

Fig. 4. - Evolution de la racine cubique de l'intensité du signal conjugué en fonction de la puissance du laser $\left(\theta=6,8^{\circ}\right)$.

[Evolution of the cubic root of the signal versus the laser power $\left(\theta=6.8^{\circ}\right)$.]

$p$ est une constante, et $R$ est alors donné par l'expression:

$$
R=\frac{2 Z}{Y-X-Z}
$$

Si les deux premiers paramètres sont immédiatement déterminés à partir de l'enregistrement, $Z$ nécessite une exploitation informatique de la décroissance exponentielle de l'amplitude du réseau de grand pas. Cette dernière nous fournit l'amplitude initiale, donc $Z$, et la constante de temps de relaxation de ce réseau, $\left(D q_{2}^{2}\right)^{-1}$, et par là le rayon hydrodynamique des vésicules puisque $D=\frac{k T}{6 \pi r \eta}$ La variation expérimentale de $R$ en fonction de $\Lambda_{2}^{2}$ est donnée en figure 5. Malgré la forte dispersion de deux points, on constate que cette variation ne présente pas de pente révélatrice d'un processus thermodiffusif pour les vecteurs d'onde explorés. Un tel processus faisant intervenir dans l'expression du rapport $R$ un terme additif en $\frac{k_{\mathrm{t}}}{q_{2}^{2}}[6]$, où $k_{\mathrm{t}}$ est le rapport thermodiffusif, de signe et de valeur inconnus pour notre suspension. Ayant par ailleurs reporté les valeurs théoriques attendues en prenant pour valeur du rayon externe des vésicules $r=49 \mathrm{~nm}( \pm 14 \mathrm{~nm})$ obtenue à partir de l'exploitation des dix décroissances exponentielles, nous constatons que les valeurs expérimentales de $R$ sont le plus souvent supérieures aux valeurs théoriques. Ce constat a été systématiquement retrouvé sur toutes les autres séries d'enregistrement réalisées avec des préparations de vésicules différentes. Ceci s'explique par le fait que les préparations ne sont pas strictement monodisperses [4]. En effet d'une part, la simulation numérique à l'aide d'une monoexponentielle appliquée sur le début de la relaxation du réseau de grand pas, évalue le rayon des particules les plus petites qui diffusent de ce fait le plus rapidement : nous sousestimons donc systématiquement $r$. D'autre part, parce que la valeur théorique de $R$, pour $r$ supposé estimé correctement, est inférieure à la valeur théorique moyenne de $R$ pour 


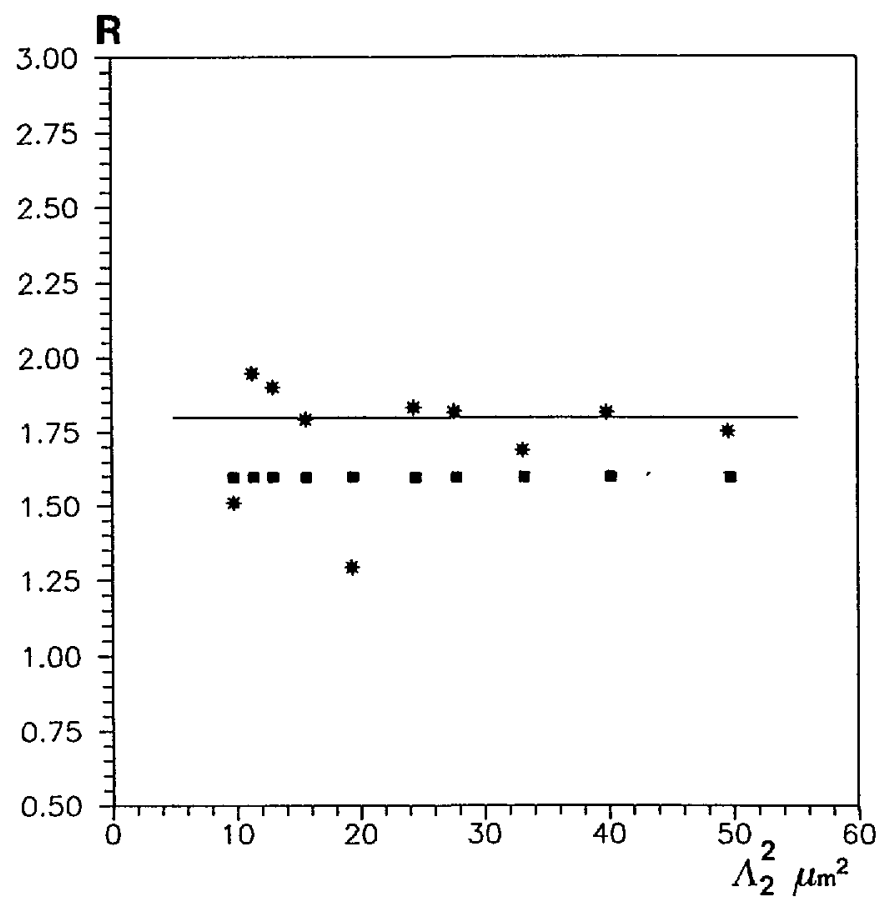

Fig. 5. - Evolution du rapport $R$ des deux contributions en fonction du carré du pas du réseau de grand pas. Les étoiles sont les valeurs expérimentales et les carrés les valeurs théoriques attendues.

[Evolution of the ratio $R$ of the two contributions as a function of the square of the coarse grating period. Stars are experimental data, squares are theoretical expected values.]

l'ensemble des tailles des particules : en effet $\overline{R(\Theta, r)}>R(\Theta, \bar{r})$, comme l'indiquent les valeurs de $R$ pour différents rayons dans le tableau suivant, valeurs qui ne croissent pas linéairement.

\begin{tabular}{|c|c|c|c|c|c|}
\hline$r(\mathrm{~nm})$ & 30 & 40 & 50 & 60 & 70 \\
\hline$R$ & 1,17 & 1,33 & 1,60 & 2.02 & 2.72 \\
\hline
\end{tabular}

Nous avons aussi comparé pour des situations expérimentales identiques $(P=3 \mathrm{~W})$, et pour une même fraction volumique, l'amplitude du signal conjugué obtenue avec une suspension de VPU par rapport à celle obtenue avec une suspension aqueuse de billes de latex de $40 \mathrm{~nm}$ de rayon, suspension souvent utilisée comme milieu de référence pour ce type de non-linéarités [11]. Si la première était de l'ordre de $0,5 \mu \mathrm{W}$, la seconde atteignait $6 \mu W$. Ceci confirme tout d'abord les valeurs importantes attendues pour l'indice de réfraction non linéaire $n_{2}$ de suspensions de VPU (en supposant un processus électrostrictif être seul à l'origine des non-linéarités). D'autre part on notera que le rapport à la puissance quatre des polarisabilités des deux types de particules se révèle bien, en première approximation, de l'ordre de grandeur du rapport des amplitudes des signaux conjugués, et ce en accord avec notre approche théorique. Par ailleurs encore, bien que les billes pleines de latex offrent des non-linéarités d'amplitude supérieure à celle des coquilles phospholipidiques, on remarquera qu'une augmentation de leur rayon les fait sortir du cadre de l'approximation de Born, ce qui n'est pas le cas pour les secondes tout en augmentant leur polarisabilité et donc les non- 
linéarités attendues. Enfin la possibilité de doper la bicouche phospholipidique, soit pour modifier la partie réelle de son indice de réfraction soit pour modifier sa partie imaginaire (colorants hydrophobes), présente un intérêt original que nous étudions actuellement.

En conclusion, pour la première fois à notre connaissance, nous avons mis en évidence des non-linéarités optiques dans une suspension de vésicules phospholipidiques unilamellaires. Les non-linéarités analysées par une expérience de conjugaison de phase sont importantes et sont d'origine électrostrictive. L'absence d'une intervention significative d'un effet thermodiffusif Soret, pour la taille choisie des vésicules et pour les conditions de notre expérience, est en accord avec l'approche théorique de cet effet faite par D. Lhuillier [9]. Ces vésicules phospholipidiques unilamellaires sont idéales pour la validité d'un traitement théorique fondé sur l'approximation de Born afin de quantifier l'amplitude des non-linéarités.

\section{Remerciements.}

Nous remercions très vivement Monsieur B. Pouligny du C.R.P.P. de Pessac pour ses interventions déterminantes dans ce travail; Madame J. Lalanne pour avoir assuré la fabrication des VPU ; Madame P. Vaca-Segond et Messieurs P. Meleard et L. Sarger pour leur aide précieuse.

\section{Bibliographie}

[1] Palmer A. J., Opt. Lett. 5 (1980) 54-55.

[2] ASHKIN A., Opt. Lett. 11 (1986) 288-290.

[3] Ashkin A., Dziedzic J. M., Yamane T., Nature 330 (1987) 769-771. ASHKIN A., DZIEDZIC J. M., Sciences 235 (1989) 1517-1520.

[4] Hope M. J., Bailly M. B., Webb G., Cullis P. R., Bioch. Biophys. Acta 812 (1985) 55-65.

[5] Cherry R. J., Chapman D., J. Mol. Biol. 40 (1969) 19-32.

[6] Freysz E., Laffon E., Ducasse A., Opt. Lett. 16 (1991) 1644-1646.

[7] Giglio M., Vendramini A., P.R.L. 38 (1977) 26-30.

[8] Jeanjean B., Freysz E., Ducasse A., Pouligny B., Europhys. Lett. 7 (1988) 219-224; Jeanjean B., Freysz E., Ponton A., Ducasse A., Pouligny B., Phys. Rev. A 39 (1989) 5268 5272 .

[9] LHUILlier D., J. Phys. 47 (1986) 1687-1696.

[10] Pecora R., Aragon S. R., Chem. Phys. Lipids 13 (1970) 1-10.

[11] SMith P. W., Ashkin A., Tomlinson W. J., Opt. Lett. 6 (1981) 284-286. 
Classification

Physics Abstracts

$02.60-87.10-87.20 \mathrm{C}$

\title{
Nearly spherical vesicle shapes calculated by use of spherical harmonics : axisymmetric and nonaxisymmetric shapes and their stability
}

\author{
V. Heinrich $\left({ }^{1}\right)$, M. Brumen $\left({ }^{2,4}\right)$, R. Heinrich $\left({ }^{1}\right)$, S. Svetina $\left({ }^{3,4}\right)$ \\ and B. Žekš $\left({ }^{3,4}\right)$ \\ (1) Institute of Biophysics, Humboldt University Berlin, Invalidenstrasse 42, 0-1040 Berlin, \\ Germany \\ (2) Department of Physics, Pedagogical Faculty, University of Maribor, Koroška cesta 160, Yu- \\ 62000 Maribor, Slovenia \\ (3) Institute of Biophysics, Medical Faculty, University of Ljubljana, Lipičeva 2, Yu-61105 \\ Ljubljana, Slovenia \\ $\left({ }^{4}\right)$ J. Stefan Institute, Jamova 39, Yu-61000 Ljubljana, Slovenia
}

(Received 15 October 1991, accepted in final form 21 January 1992)

Résumé. - On développe une approche théorique pour déterminer la forme quasi sphérique des vésicules phospholipidiques. La méthode est générale, dans le sens qu elle ne dépend pas d'une quelconque restriction de symétrie. On suppose que la forme à l'équilibre correspond au minimum d'énergie de tension de courbure de la membrane pour une valeur constante de la surface de la membrane, du volume des vésicules et de la différence de surface des doubles feuillets phospholipidiques de la membrane. L'énergie de courbure et les contraintes sont développées à l'ordre 4 en terme de la déviation de la forme par rapport à une sphère. Tous les termes jusqu'au troisième ordre sont inclus dans les calculs suivants. La déviation est exprimée en séries d'harmoniques sphériques. On montre que la stabilité des solutions peut être testée en regardant les valeurs propres de la matrice des dérivées secondes de l'énergie de courbure par rapport aux amplitudes indépendantes du développement en harmoniques sphériques. La méthode est appliquée aux calculs des formes à symétrie axiale ou non, et les influences des différentes approximations sont étudiées. On montre que pour des variations de la différence dans l'aire des feuillets, on peut transformer de manière continue une forme stable aplatie en une forme stable allongée et, réciproquement.

Abstract. - A theoretical approach to determine nearly spherical shapes of phospholipid vesicles
is developed. The method is general in the sense that it does not depend on any symmetry
restrictions. Equilibrium shapes are assumed to correspond to the minimum of the membrane
bending elastic energy at constant values of the membrane area, the vesicle volume and the
difference of areas of the two leaflets of the phospholipid bilayer. The bending energy and the
constraints are expanded up to fourth order terms in the deviation from a sphere, and in the
subsequent calculations all terms up to the third order are included. The deviation is expressed as
a series of spherical harmonics. It is shown that the stability of the solutions can be tested by
inspecting the eigenvalues of the matrix of second derivatives of the bending energy with respect
to independent amplitudes of spherical harmonics expansion. The method is applied to the 
calculation of axisymmetric and nonaxisymmetric shapes, and the influences of different approximations are discussed. It is shown that at variations of the leaflet area difference stable oblate and stable prolate shapes are transformed into each other in a continuous manner.

\section{Introduction.}

Phospholipid vesicles in flaccid conditions, i.e. when their volume is smaller than the volume of a sphere of the same membrane area, are known to exhibit a broad variety of different shapes. Several phenomena connected with shape transformations of vesicles as well as corresponding experimental and theoretical investigations were recently reviewed by Lipowsky [1].

In general, theoretical concepts on vesicle shapes are based on the assumption that equilibrium states correspond to the minimum of the membrane bending energy. Introducing the spontaneous curvature Helfrich [2] formulated an expression for the bending energy which has been widely used to determine equilibrium shapes of vesicles at given values of the vesicle volume and the membrane area $[3,4]$. Svetina and Žekš $[5,6]$ worked out a slightly different concept of calculating vesicle shapes. In this concept the constraint of constant membrane area of the vesicle was extended in such a way that it was applied to both leaflets of the phospholipid bilayer, i.e. it was assumed that under equilibrium conditions the areas of both monolayers are fixed. Since this theoretical concept can explain shape changes in accordance with the bilayer couple hypothesis [7] it is called the bilayer couple model.

Both models, the spontaneous curvature concept as well as the bilayer couple model, have been widely studied by applying an Euler-Lagrange ansatz to the resulting variational problem [2-6]. Recently, the results of both approaches were compared on the basis of a detailed investigation of the corresponding phase diagrams [8]. Up to now, the computations using this ansatz have been restricted to axisymmetric shapes, and a complete stability analysis of the resulting shapes has not been performed.

The investigation of the bilayer couple model by the use of an Euler-Lagrange ansatz revealed that the axisymmetric shapes obtained can be assigned to different classes [9]. A given class comprises all shapes of the same symmetry which can be continuously transformed into each other changing the model parameters. The axisymmetric shapes have been characterized in more detail for the sake of some specific studies: the bilayer couple interpretation of shape transformations of red blood cells [6], the investigation of cell polarity $[9,10]$, and the interpretation of temperature induced shape transformations of giant vesicles [11]. In the latter work as well as in [12] a good agreement of experimental and theoretical results was found.

In order to extend the theoretical investigation by including also nonaxisymmetric shapes, in the present paper another method is studied which is based on a Taylor expansion of the membrane bending energy with respect to the deviation from the spherical shape. Subsequently, the deviation is expressed as a series of spherical harmonics. Using a Ritz procedure (cf. [16]) those amplitudes of spherical harmonics are calculated which minimize the bending energy taking into account the various constraints. A necessary condition to obtain equilibrium states is that the first derivatives of the corresponding functional with respect to amplitudes of spherical harmonics vanish. In addition, the method enables the stability analysis of a given solution by deciding whether the bending energy has a minimum with respect to variations of the amplitudes. For that one has to inspect the eigenvalues of the matrix of second derivatives of the bending energy with respect to independent amplitudes. A shape is stable if all eigenvalues of this matrix are positive. 
Due to the expansion in terms of the displacement from a sphere the method requires to restrict the calculations to nearly spherical shapes. Such a Taylor expansion has been worked out by Helfrich [13] up to the second order terms, and the results have been widely used in the analysis of thermal fluctuations of vesicle shapes $[14,15]$. In the present work the bending energy as well as the constraints are expanded up to the fourth order terms. Into the subsequent computations all terms of the deviation up to the third order are included. Third order calculations have been carried out before using the spontaneous curvature concept $[17,18]$. However, in these works only special questions were considered by applying the general equations obtained. Third order terms have been analysed also in [19] but without calculation of equilibrium shapes since the volume was assumed to change due to external pressure changes. Hitherto, the bilayer couple model has not been analysed in this way. Various nonaxisymmetric vesicle shapes were discussed in $[17,19]$ as well as in $[20,21]$. However, a systematic and explicit determination of equilibrium shapes of any symmetry including a complete analysis of their stability is still lacking.

In the following, vesicle shapes will be considered within the bilayer couple model. Accordingly, for the membrane bending energy the expression given in [6] is used. Equilibrium shapes are calculated by minimizing the bending energy at constant areas of both leaflets of the phospholipid bilayer or, more conveniently, at a constant area of the inner monolayer $(A)$ and at a constant difference between the areas of the two layers $(\Delta A)$. Furthermore, the volume $V$ of the vesicle is assumed to be constant because water transport through phospholipid membranes is known to be very slow during the relevant times of observing equilibrium shapes.

The paper is organized as follows. In sections 2-4 the general equations for the calculation of nearly spherical shapes of any symmetry are derived. In section 5 a method for the stability analysis is developed. In section 6 the general model equations are specified for axisymmetric shapes. The results of this special case are compared with those of an Euler-Lagrange ansatz in section 7. In section 8 the effects of different approximations of the present method are studied. Subsequently, the model is applied in its general form and nonaxisymmetric shapes are calculated including the analysis of their stability (Sect. 9). In the numerical computations the maximal $\ell$-value used in the series of spherical harmonics $Y_{\ell_{m}}$ is so high that the inclusion of functions $Y_{\ell_{m}}$ with higher $\ell$ would not significantly change the results. Concerning the determination of stable equilibrium shapes the method is, therefore, more general than those used in previous works $[17,18]$ where only $\ell=2$ deformations of the sphere have been considered.

\section{The model.}

Equilibrium shapes of phospholipid vesicles are assumed to be characterized by the minimal value of the membrane bending energy

$$
W_{\mathrm{b}}=\frac{1}{2} k_{\mathrm{c}} \int\left(c_{1}+c_{2}\right)^{2} \mathrm{~d} A
$$

where $k_{\mathrm{c}}$ is the membrane bending elastic constant, $c_{1}$ and $c_{2}$ are the two principal curvatures, and integration is performed over the closed surface of the inner monolayer. The shapes have to fulfil the conditions

$$
\begin{aligned}
A & =A_{0} \\
V & =V_{0} \\
\Delta A & =\Delta A_{0}
\end{aligned}
$$


i.e. the membrane area $(A)$, cell volume $(V)$ and the difference between the areas of the two membrane leaflets $(\Delta A)$ are considered to be fixed at values $A_{0}, V_{0}$, and $\Delta A_{0}$, respectively. The difference in leaflet areas is given by the formula

$$
\Delta A=h \int\left(c_{1}+c_{2}\right) \mathrm{d} A
$$

where $h$ is the distance between the two leaflets (cf. [22]). In equation (5) a second order term with respect to $h$ is neglected since the distance between the two monolayers is very small in the relevant length scale.

The closed surface representing the vesicle shape can be described by $\tilde{r}=\tilde{r}(\vartheta, \varphi)$ using the spherical angles $\vartheta$ and $\varphi$ as independent coordinates. $\tilde{r}$ is the distance between the origin of the coordinate system and the surface of the vesicle. It must be required that $\tilde{r}=\tilde{r}(\vartheta, \varphi)$ is a unique function of $\vartheta$ and $\varphi$.

For the following derivations dimensionless quantities are introduced. The bending energy $\left(W_{\mathrm{b}}\right)$ and the constraints $(A, V$ and $\Delta A)$ are normalized relative to those values they would attain if, at the given surface area $A_{0}$, the shape were spherical with radius $R_{s}=\left(A_{0} / 4 \pi\right)^{1 / 2}$ This normalization procedure is the same as used in [6]. The dimensionless model quantities are identified by small letters $w_{\mathrm{b}}, a, v$ and $\Delta a$, respectively. Correspondingly, the shape function $\tilde{r}(\vartheta, \varphi)$ has to be normalized relative to $R_{s}$ which yields the dimensionless shape function $r(\vartheta, \varphi)=\tilde{r}(\vartheta, \varphi) / R_{s}$. Obviously, conditions (2)-(4) then read in a dimensionless form as follows : $a=a_{0}=1, v=v_{0}$ and $\Delta a=\Delta a_{0}$, respectively.

Taking into account the relations between Cartesian and spherical coordinates as well as the definitions of the coefficients of the first and the second fundamental form of the surface it is possible to express the bending energy, the membrane area, the cell volume as well as the difference between the areas of the two membrane leaflets in terms of $r, \vartheta$ and $\varphi$. The resulting equations read in a dimensionless form :

$$
\begin{aligned}
w_{\mathrm{b}} & =\frac{1}{16 \pi} \int\left[2 r-\Delta r+\frac{r[\nabla r]^{2}+\frac{1}{2} \nabla r \cdot \nabla\left([\nabla r]^{2}\right)}{r^{2}+[\nabla r]^{2}}\right]^{2} \frac{\mathrm{d} \Omega}{r \sqrt{r^{2}+[\nabla r]^{2}}} \\
a & =\frac{1}{4 \pi} \int r \sqrt{r^{2}+[\nabla r]^{2}} \mathrm{~d} \Omega \\
v & =\frac{1}{4 \pi} \int r^{3} \mathrm{~d} \Omega \\
\Delta a & =\frac{1}{8 \pi} \int\left[2 r-\Delta r+\frac{r[\nabla r]^{2}+\frac{1}{2} \nabla r \cdot \nabla\left([\nabla r]^{2}\right)}{r^{2}+[\nabla r]^{2}}\right] \mathrm{d} \Omega .
\end{aligned}
$$

Integration is performed over the full solid angle $\mathrm{d} \Omega=\sin \vartheta \mathrm{d} \vartheta \mathrm{d} \varphi$. The differential operators $\nabla$ and $\Delta$ are adopted, respectively, as

$$
\begin{aligned}
& \nabla=\left(\frac{\partial}{\partial \vartheta}, \frac{1}{\sin \vartheta} \frac{\partial}{\partial \varphi}\right) \\
& \Delta=\frac{\partial^{2}}{\partial \vartheta^{2}}+\frac{\cos \vartheta}{\sin \vartheta} \frac{\partial}{\partial \vartheta}+\frac{1}{\sin ^{2} \vartheta} \frac{\partial^{2}}{\partial \varphi^{2}}
\end{aligned}
$$


The function $r=r(\vartheta, \varphi)$ can be written as the sum of a certain constant $r_{0}$ and a function $u(\vartheta, \varphi)$ :

$$
r(\vartheta, \varphi)=r_{0}+u(\vartheta, \varphi)
$$

$r_{0}$ is the radius of a sphere which is called the corresponding sphere. Consequently, $u(\vartheta, \varphi)$ is the deviation from this sphere. For nearly spherical shapes the radius of the corresponding sphere $r_{0}$ can always be chosen in such a way that for any values of $\vartheta$ and $\varphi$ the displacement $u(\vartheta, \varphi)$ is much smaller than $r_{0}$. Using relation (12) in equations (6-9) and expanding the resulting expressions up to the fourth order in the relative displacement $u / r_{0}$ one obtains :

$$
\begin{aligned}
& w_{\mathrm{b}}=1+\frac{1}{16 \pi r_{0}^{4}} \int\left[-4 r_{0}^{3} \Delta u+4 r_{0}^{2} u \Delta u+r_{0}^{2}(\Delta u)^{2}+2 r_{0}^{2}[\nabla u]^{2}-\right. \\
& -4 r_{0} u^{2} \Delta u-2 r_{0} u(\Delta u)^{2}-4 r_{0} u[\nabla u]^{2}+2 r_{0} \nabla \mathrm{u} \cdot \nabla\left([\nabla u]^{2}\right)+ \\
& +\left\{4 u^{3} \Delta u+3 u^{2}(\Delta u)^{2}+6 u^{2}[\nabla u]^{2}-6 u \nabla u \cdot \nabla\left([\nabla u]^{2}\right)-\right. \\
& \left.\left.-\frac{1}{2}(\Delta u)^{2}[\nabla u]^{2}-\Delta u \nabla u \cdot \nabla\left([\nabla u]^{2}\right)-\frac{7}{2}[\nabla u]^{4}\right\}\right] \mathrm{d} \Omega \\
& a=r_{0}^{2}+\frac{1}{4 \pi r_{0}^{2}} \int\left[2 r_{0}^{3} u+r_{0}^{2} u^{2}+\frac{1}{2} r_{0}^{2}[\nabla u]^{2}+\left\{-\frac{1}{8}[\nabla u]^{4}\right\}\right] \mathrm{d} \Omega \\
& v=r_{0}^{3}+\frac{3}{4 \pi} \int\left[r_{0}^{2} u+r_{0} u^{2}+\frac{1}{3} u^{3}\right] \mathrm{d} \Omega \\
& \Delta a=r_{0}+\frac{1}{8 \pi r_{0}^{3}} \int\left[2 r_{0}^{3} u-r_{0}^{3} \Delta u+r_{0}^{2}[\nabla u]^{2}-r_{0} u[\nabla u]^{2}+\frac{1}{2} r_{0} \nabla u \cdot \nabla\left([\nabla u]^{2}\right)+\right. \\
& \left.+\left\{u^{2}[\nabla u]^{2}-u \nabla \mathrm{u} \cdot \nabla\left([\nabla u]^{2}\right)-[\nabla u]^{+}\right\}\right] \mathrm{d} \Omega .
\end{aligned}
$$

The further treatment of fourth order terms (marked by the $\{\ldots\}$-brackets) turned out to be a very complex and extensive procedure. Therefore, the present model is restricted to terms up to the third order in $u$, i.e. all fourth order terms are neglected. Note that in the following any quotation of equations (13-16) refers to the third order versions of these equations not taking into account the terms enclosed by curved brackets.

The integrals needed in equations (13-16) can be calculated by expressing the displacement $u$ as a series of spherical harmonics :

$$
u(\vartheta, \varphi)=\sum_{\ell=0}^{\infty} \sum_{m=-\ell}^{\ell} U_{\ell_{m}} Y_{\ell m}(\vartheta, \varphi) .
$$

The inclusion of the term $U_{00} Y_{00}$ permits to fix the radius $r_{0}$ of the corresponding sphere without loosing the dependence of the solutions on a certain constant part of $r(\vartheta, \varphi)$. Here, $r_{0}$ is chosen in such a way that the corresponding sphere has the same volume as the vesicle, i.e. in normalized quantities $r_{0}=\left(v_{0}\right)^{1 / 3}$

Since the displacement $u$ is real the amplitudes have to obey the relation

$$
U_{\ell m}^{*}=(-1)^{m} U_{\ell,-m}
$$

The spherical harmonics $Y_{\ell_{m}}(\vartheta, \varphi)$ are expressed by the associated Legendre polynomials $P_{\ell_{m}}(\cos \vartheta)$ as

$$
Y_{\ell_{m}}(\vartheta, \varphi)=\sqrt{\frac{2 \ell+1}{4 \pi} \cdot \frac{(\ell-m) !}{(\ell+m) !}} P_{\ell_{m}}(\cos \vartheta) \mathrm{e}^{\imath m \varphi}
$$


After introducing expansion (17) into equations (13-16) the corresponding integrals over the closed surface may be calculated. Basic properties of spherical harmonics, types of integrals as well as details of the calculations are given in the Appendix. As a final result the expressions for the bending energy, membrane area, volume and difference between areas of the two membrane leaflets, respectively, attain the following form

$$
\begin{gathered}
w_{\mathrm{b}}=1+\frac{1}{16 \pi r_{0}^{2}} \sum_{\ell=0}^{\infty} \ell(\ell+1)(\ell(\ell+1)-2) U_{\ell}^{(2)}+ \\
\quad+\frac{1}{8 \pi r_{0}^{3}} \sum_{\ell_{1}} \sum_{\ell_{2}} \sum_{\ell_{3}} \ell_{1}\left(\ell_{1}+1\right)\left(-\frac{\ell_{1}\left(\ell_{1}+1\right)}{2}+1\right) U_{\ell_{1}, \ell_{2}, \ell_{3}}^{(3)} \\
a=r_{0}^{2}+\frac{2 r_{0}}{\sqrt{4 \pi}} U_{00}+\frac{1}{4 \pi} \sum_{\ell=0}^{\infty}\left(\frac{\ell(\ell+1)}{2}+1\right) U \ell_{\ell}^{(2)} \\
v=r_{0}^{3}+\frac{3 r_{0}^{2}}{\sqrt{4 \pi}} U_{00}+\frac{3 r_{0}}{4 \pi} \sum_{\ell=0}^{\infty} U(2)+\frac{1}{4 \pi} \sum_{\ell_{1}} \sum_{\ell_{2}} \sum_{\ell_{3}} U_{\ell_{1}, \ell_{2}, \ell_{3}}^{(3)} \\
\Delta a=r_{0}+\frac{1}{\sqrt{4 \pi}} U_{00}+\frac{1}{8 \pi r_{0}} \sum_{\ell=0}^{\infty} \ell(\ell+1) U_{\ell}^{(2)}+ \\
+\frac{1}{16 \pi r_{0}^{2}} \sum_{\ell_{1}} \sum_{\ell_{2}} \sum_{\ell_{3}} \ell_{1}\left(\ell_{1}+1\right)\left(-\frac{\ell_{1}\left(\ell_{1}+1\right)}{2}+\ell_{2}\left(\ell_{2}+1\right)-1\right) U_{\ell_{1}, \ell_{2}, \ell_{3}}^{(3)}
\end{gathered}
$$

where

$$
\begin{aligned}
U_{\ell}^{(2)} & =\sum_{m=-\ell}^{\ell} U_{\ell_{m}} U_{\ell_{m}}^{*} \\
U_{\ell_{1}, \ell_{2}, \ell_{3}}^{(3)} & =\sum_{m_{1}=-\ell_{1} m_{2}=-\ell_{2}}^{\ell_{1}} \sum_{\ell_{1} m_{1}}^{\ell_{2}} U_{\ell_{2} m_{2}} U_{\ell_{3}, m_{1}+m_{2}}^{*} A\left(\ell_{1} \ell_{2} \ell_{3} ; m_{1} m_{2}\right) .
\end{aligned}
$$

Coefficients $A\left(\ell_{1} \ell_{2} \ell_{3} ; m_{1} m_{2}\right)$ are given by equation (A2) in the Appendix. Summations in equations $(20-25)$ have to be performed under the following conditions :

$$
\begin{aligned}
& \left|\ell_{1}-\ell_{2}\right| \leqslant \ell_{3} \leqslant \ell_{1}+\ell_{2} \\
& \left|m_{i}\right| \leqslant \ell_{1}, \quad i=1,2 \\
& \left|m_{1}+m_{2}\right| \leqslant \ell_{3} .
\end{aligned}
$$

Obviously, the relations (27) and (28) include the conditions $0 \leqslant \ell,(i=1,2,3)$ whereas there is no upper limit for the $\ell_{I}$.

\section{Position of the coordinate system.}

There is no interest in calculating the same equilibrium shape in different reference frames where the various states can be transformed into each other by translation or rotation. Therefore, one has to choose a suitable position for the origin of the coordinate system as well as for the directions of the coordinate axes. This is done here by introducing the following additional requirements :

(i) The mass centre of the vesicle $\left(\tilde{x}_{m}, \tilde{y}_{m}, \tilde{z}_{m}\right)$ is the origin of the coordinate system.

(ii) The $z$-axis $(\vartheta=0)$ of the Cartesian coordinate system has the same direction as the surface normal at the point $r(0, \varphi)$. 
(iii) The $x$-axis $(\vartheta=\pi / 2, \varphi=0$ ) is chosen in such a way that for $\varphi=0$ the function $r(\pi / 2, \varphi)$ has an extremum with respect to varying values of $\varphi$.

The first requirement yields three conditions which have to be satisfied while minimizing the bending energy :

$$
\begin{aligned}
& x_{m}=\frac{3}{16 \pi v_{0}} \int\left(r_{0}+u\right)^{4} \sin \vartheta \cos \varphi \mathrm{d} \Omega=0 \\
& y_{m}=\frac{3}{16 \pi v_{0}} \int\left(r_{0}+u\right)^{4} \sin \vartheta \sin \varphi \mathrm{d} \Omega=0 \\
& z_{m}=\frac{3}{16 \pi v_{0}} \int\left(r_{0}+u\right)^{4} \cos \vartheta \mathrm{d} \Omega=0 .
\end{aligned}
$$

Equations (29-31) were transformed into a dimensionless form by the above mentioned normalization procedure. Correspondingly, the dimensionless coordinates of the mass centre of the vesicle are defined as follows: $x_{m}=\tilde{x}_{m} / R_{\mathrm{s}}, y_{m}=\tilde{y}_{m} / R_{\mathrm{s}}$ and $z_{m}=\tilde{z}_{m} / R_{\mathrm{s}}$. Taking into account only terms up to the third order in the deviation $u$ the various integrals in equations (29-31) are calculated after the introduction of spherical harmonics (Eq. (17)). The results are also given in the Appendix (Eqs. (A18-A27)).

The second requirement is identical with the condition

$$
\left.\frac{\partial r(\vartheta, \varphi)}{\partial \vartheta}\right|_{\vartheta=0}=\left.\frac{\partial u(\vartheta, \varphi)}{\partial \vartheta}\right|_{\vartheta=0}=0
$$

which must be fulfilled at the surface point $r(\vartheta=0, \varphi)$ for any value of $\varphi$. After introducing the expansion (17) as well as the definition (19) the derivatives of the associated Legendre polynomials with respect to $\vartheta$ have to be calculated for $\vartheta=0$. It can easily be proved that these derivatives assume the following values:

$$
\left.\frac{\partial P_{\ell m}(\cos \vartheta)}{\partial \vartheta}\right|_{\vartheta=0}=\left\{\begin{array}{cl}
\frac{1}{2} & \text { if } m=-1 \\
-\frac{\ell(\ell+1)}{2} & \text { if } m=1 \\
0 & \text { else . }
\end{array}\right.
$$

Using this property together with relation (18) and considering the real and the imaginary part of the resulting expression separately one obtains from equation (32) the following two constraints :

$$
\begin{aligned}
& s_{1}=\sum_{\ell=1}^{\infty} \sqrt{\frac{2 \ell+1}{4 \pi} \ell(\ell+1)}\left(U_{\ell 1}+U_{\ell 1}^{*}\right)=0 \\
& s_{2}=i \sum_{\ell=1}^{\infty} \sqrt{\frac{2 \ell+1}{4 \pi} \ell(\ell+1)}\left(U_{\ell_{1}}-U_{\ell 1}^{*}\right)=0 .
\end{aligned}
$$

The third requirement yields the condition

$$
\left.\frac{\partial r(\vartheta, \varphi)}{\partial \varphi}\right|_{\vartheta=\frac{\pi}{2}, \varphi=0}=\left.\frac{\partial u(\vartheta, \varphi)}{\partial \varphi}\right|_{\vartheta=\frac{\pi}{2}, \varphi=0}=0 .
$$

Using equations (17) and (19) and taking into account the relations between $P_{\ell m}$ and $P_{P,-m}$ as well as equation (18) one obtains the constraint :

$$
s_{3}=i \sum_{\ell=1}^{\infty} \sum_{m=1}^{\ell} m \sqrt{\frac{2 \ell+1}{4 \pi} \cdot \frac{(\ell-m) !}{(\ell+m) !}} P_{\ell_{m}}(0)\left(U_{\ell m}-U_{\ell m}^{*}\right)=0 .
$$




\section{Calculation of equilibrium shapes.}

The shape of a vesicle can be determined by minimizing the following function containing the bending energy and all constraints in a dimensionless form

$$
\begin{aligned}
g=w_{\mathrm{b}}+\lambda_{a}(a-1)+\lambda_{v}\left(v-v_{0}\right)+ & \lambda_{\Delta}\left(\Delta a-\Delta a_{0}\right)+ \\
& +\lambda_{x} x_{m}+\lambda_{y} y_{m}+\lambda_{z} z_{m}+\lambda_{s_{1}} s_{1}+\lambda_{s_{2}} s_{2}+\lambda_{s_{3}} s_{3} .
\end{aligned}
$$

The $\lambda$ 's are Lagrange multipliers associated with the nine quantities which are fixed by the constraints. Replacing $w_{\mathrm{b}}, a, v, \Delta a, x_{m}, y_{m}, z_{m}, s_{1}, s_{2}$ and $s_{3}$ in equation (38) by the corresponding expressions given in equations (20-25), (29-31) (together with (A18-A27), cf. Appendix, and neglecting the $u^{4}$-term), (34), (35) and (37), $g$ may be written as a function of the amplitudes $U_{\ell_{m}}$ as well as of the nine Lagrange multipliers. According to Ritz' procedure, at equilibrium partial derivatives of $g$ with respect to these unknown variables must vanish. This yields a system of equations from which the amplitudes and the values of Lagrange multipliers can be obtained. Since this equation system is nonlinear its solutions can not be calculated explicitely but may be obtained numerically, for example, by the use of Newton's method.

For numerical computations it is more convenient to use real amplitudes of spherical harmonics instead of the complex ones. This is possible because all quantities necessary to determine the equilibrium shapes are real. In this work real amplitudes $X_{\ell_{m}}^{r}$ and $X_{\ell_{m}}^{i}$ are introduced by the following substitution:

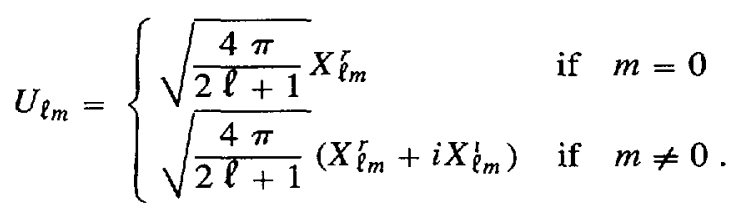

Taking into account equation (18) one obtains for $m \neq 0$ the following relations between the real amplitudes :

$$
\begin{aligned}
& X_{\ell,-m}^{r}=(-1)^{m} X_{\ell_{m}}^{r} \\
& X_{\ell,-m}^{!}=-(-1)^{m} X_{\ell_{m}}^{l} .
\end{aligned}
$$

It can easily be proved that using equation (39) all expressions containing complex amplitudes $U_{\ell_{m}}$ may be rewritten in such a way that only real amplitudes appear and the imaginary parts of all expressions vanish.

After all, the method to describe nearly spherical vesicle shapes is complete. Numerical computations require a restriction to a finite number of spherical harmonics which means that the infinite series of spherical harmonics has to be cut at an appropriately chosen maximal value $\ell_{\max }$. The numerical problem is then to determine the values of $\left(\ell_{\max }+1\right)^{2}$ unknown amplitudes together with nine unknown Lagrange multipliers.

\section{Stability analysis.}

A shape obtained as described above is stable if the extremum of the bending energy is a minimum with respect to variations of the amplitudes of spherical harmonics. Since this extremum was calculated in the presence of constraints it is necessary to distinguish between dependent and independent variables. From the whole set of amplitudes the dependent ones can be chosen arbitrarily but taking into account that their number is fixed by the number of 
constraints, and that the matrix of derivatives of the constraints with respect to dependent variables must be regular. Let $n$ denote the total number of amplitudes and $m(0 \leqslant m<n)$ the number of dependent ones. Then independent and dependent amplitudes may be represented by the components $x_{i}(i=1, \ldots, n-m)$ and $y_{k}(k=1, \ldots, m)$ of vectors $\mathbf{x}$ and $\mathbf{y}$, respectively. The functional dependence of the bending energy on amplitudes shall be denoted by $f(\mathbf{x}, \mathbf{y})$. Taking into consideration the relations given by the constraints one may write

$$
f^{*}(\mathbf{x})=f(\mathbf{x}, \mathbf{y}(\mathbf{x})) \text {. }
$$

An extremum of $f^{*}$ is a minimum if all eigenvalues of the matrix of second derivatives of $f^{*}$ with respect to independent variables, $\partial^{2} f^{*} / \partial x_{i} \partial x_{j}(i, j=1, \ldots, n-m)$, are positive. The elements of this matrix may be determined in the following way. First and second derivatives of $f^{*}$ with respect to independent variables $x_{i}$ and $x_{j}(i, j=1, \ldots, n-m)$ read

$$
\begin{aligned}
& \frac{\partial f^{*}}{\partial x_{i}}= \sum_{k=1}^{m} \frac{\partial f}{\partial y_{k}} \cdot \frac{\partial y_{k}}{\partial x_{i}}+\frac{\partial f}{\partial x_{i}} \\
& \frac{\partial^{2} f^{*}}{\partial x_{\imath} \partial x_{j}}=\sum_{k=1}^{m} \frac{\partial f}{\partial y_{k}} \cdot \frac{\partial^{2} y_{k}}{\partial x_{i} \partial x_{j}}+\sum_{k=1}^{m} \sum_{\ell=1}^{m} \frac{\partial^{2} f}{\partial y_{k} \partial y_{\ell}} \cdot \frac{\partial y_{k}}{\partial x_{\imath}} \cdot \frac{\partial y_{\ell}}{\partial x_{j}}+ \\
& \quad+\sum_{k=1}^{m}\left(\frac{\partial^{2} f}{\partial y_{k} \partial x_{l}} \cdot \frac{\partial y_{k}}{\partial x_{j}}+\frac{\partial^{2} f}{\partial y_{k} \partial x_{j}} \cdot \frac{\partial y_{k}}{\partial x_{i}}\right)+\frac{\partial^{2} f}{\partial x_{i} \partial x_{j}}
\end{aligned}
$$

First and second derivatives of dependent variables with respect to independent ones are still unknown since the nonlinearities of the constraints (Eqs. (21-23) and (29-31)) do not allow an explicit formulation of the dependences $y_{k}\left(x_{t}\right)$. Their values can be obtained, however, by implicit differentiation of the equations of the constraints with respect to independent variables. From that one obtains in a first step for each $x_{i}(i=1, \ldots, n-m)$ a system of linear equations to determine the values of $\partial y_{k} / \partial x_{i}(k=1, \ldots, m)$. Repeated implicit differentiation of the corresponding equations yields systems of linear equations for the calculation of the values of all derivatives $\partial^{2} y_{k} / \partial x_{i} \partial x_{j}$ which then may be used in equation (44). A vesicle shape is stable if the extremum of $f^{*}$ is a minimum, i.e. if all eigenvalues of the matrix $\partial^{2} f * / \partial x_{i} \partial x_{j}$ are positive.

\section{Specification of the model for the axisymmetric case.}

The numerical treatment of the general method developed above is rather extensive and by far not trivial. Corresponding computations are much simpler for axisymmetric shapes than for shapes of arbitrary symmetry. The main reason is that axisymmetric shapes may be characterized by a relatively low number of amplitudes of spherical harmonics. If the maximal $\ell$-value used in the computations is denoted by $\ell_{\text {max }}$, one has to calculate in the general case $\left(\ell_{\max }+1\right)^{2}$ unknown amplitudes whereas for axisymmetric shapes this number is reduced to $\ell_{\max }+1$. Since in the axisymmetric case the coordinate system is fixed by preventing its shift along the symmetry axis the number of corresponding constraints is also reduced. Therefore, the restriction to axisymmetric vesicle shapes provides a relatively simple opportunity to study the method described above. An even more compelling argument for testing this model first by considering only axisymmetric shapes is that in this case the results can be compared with those obtained before by solving a system of Euler differential equations $[6,8]$. The latter method permits to calculate axisymmetric vesicle shapes without the approximations used by the former approach. Consequently, it is possible to evaluate the effects of the approximations of the method presented here and, therefore, its validity by comparing both methods in the 
special case of axisymmetric shapes. In this section the general expressions derived above are written for this special case.

For axisymmetric shapes the deviation $u$ from the corresponding sphere depends only on the azimuthal angle $\vartheta$ and can be expressed by the Legendre polynomials $P_{\ell}(\cos \vartheta)$ as

$$
u(\vartheta)=\sum_{\ell=0}^{\ell_{\max }} X_{\ell} P_{\ell}(\cos \vartheta)
$$

where the notation $X_{\ell}$ has been introduced for the amplitudes $X_{\ell_{0}}^{r}$ defined in (39). Amplitudes describing nonaxisymmetric features of the vesicle shapes must vanish :

$$
U_{\ell m}=0 \quad \text { if } \quad m \neq 0 .
$$

The following expressions used in equations (20-23) read in the axisymmetric case (cf. Eqs. (24) and (25)) :

$$
\begin{aligned}
U_{00} & =\sqrt{4 \pi} X_{0} \\
U_{\ell}^{(2)} & =\frac{4 \pi}{2 \ell+1} X_{\ell}^{2} \\
U_{\ell_{1}, \ell_{2}, \ell_{3}}^{(3)} & =\frac{4 \pi}{2 \ell_{3}+1} C\left(\ell_{1} \ell_{2} \ell_{3} ; 00\right)^{2} X_{\ell_{1}} X_{\ell_{2}} X_{\ell_{3}} .
\end{aligned}
$$

The $C\left(\ell_{1} \ell_{2} \ell_{3} ; 00\right)$ are Clebsch-Gordan coefficients (cf. [23]). For the summation indices condition (26) must hold, whereas the relations (27) and (28) yield the condition

$$
0 \leqslant \ell_{l} \leqslant \ell_{\max } \quad(i=1,2,3) .
$$

The requirement that the mass centre of an axisymmetric vesicle is the origin of the coordinate system is satisfied if $z_{m}$ defined in equation (31) becomes zero. Introducing the amplitudes $X_{\ell}$ the integrals needed in equation (31) (cf. (A20), (A23) and (A26)) can be rewritten, so that one obtains :

$$
\begin{aligned}
z_{m}=\frac{r_{0}}{v_{0}}\left[r_{0}^{2} X_{1}\right. & +9 r_{0} \sum_{\ell=1}^{\ell_{\max }} \frac{\ell}{(2 \ell-1)(2 \ell+1)} X_{\ell-1} X_{\ell}+3 \sum_{\ell_{1}} \sum_{\ell_{2}} \sum_{\ell_{3}} \frac{X_{\ell_{1}} X_{\ell_{2}} X_{\ell_{3}}}{2 \ell_{3}+1} \times \\
& \left.\times\left(\frac{\ell_{3}+1}{2 \ell_{3}+3} C\left(\ell_{1} \ell_{2} \ell_{3}+1 ; 00\right)^{2}+\frac{\ell_{3}}{2 \ell_{3}-1} C\left(\ell_{1} \ell_{2} \ell_{3}-1 ; 00\right)^{2}\right)\right] .
\end{aligned}
$$

The summation indices $\ell_{i}(i=1,2,3)$ have to fulfil the condition (50). Furthermore, the Clebsch-Gordan coefficients $C\left(\ell_{1} \ell_{2} \ell ; 00\right)$ with $\ell=\ell_{3}-1$ or $\ell=\ell_{3}+1$ are zero if one of the relations

$$
\begin{gathered}
\left|\ell_{1}-\ell_{2}\right| \leqslant \ell \leqslant \ell_{1}+\ell_{2} \\
0 \leqslant \ell
\end{gathered}
$$

is not satisfied.

Obviously, for axisymmetric shapes the three conditions introduced in order to prevent a rotation of the coordinate system (Eqs. (34), (35) and (37)) are always fulfilled.

Corresponding to equation (38) axisymmetric shapes are calculated minimizing the function

$$
g=w_{\mathrm{b}}+\lambda_{a}(a-1)+\lambda_{v}\left(v-v_{0}\right)+\lambda_{\Delta}\left(\Delta a-\Delta a_{0}\right)+\lambda_{z} z_{m} .
$$


After substituting $w_{\mathrm{b}}, a, v, \Delta a$ and $z_{m}$ in equation (54) by the corresponding expressions extrema of $g$ are determined for vanishing values of the first derivatives of $g$ with respect to the amplitudes $X_{0}, X_{1}, \ldots, X_{\ell_{\max }}$ as well as to the Lagrange multipliers $\lambda_{a}, \lambda_{v}, \lambda_{\Delta}$ and $\lambda_{z}$. The resulting system of nonlinear equations for the calculation of amplitudes and Lagrange multipliers is solved by the use of Newton's method. The stability of the shapes is determined as described in section 5 .

\section{Results of the axisymmetric case.}

In the following the results of the calculation of axisymmetric vesicle shapes by the use of the model equations specified in the previous section will be presented. In order to evaluate the accuracy of the model, these results are compared with those obtained before by another method described in [6]. By the latter method axisymmetric equilibrium shapes are determined on the basis of an Euler-Lagrange ansatz, i.e. without the approximations used in the model presented here. In order to distinguish between these two different approaches they are called in the following the third order method and the Euler method, respectively. The aim of this paper is to investigate in detail the third order method. Therefore, it is of special interest whether this method can reproduce the main features of the axisymmetric shapes obtained by the Euler method (cf. [6, 8]).

First, several results obtained by the Euler method shall be shortly repeated and explained. Figure 1 shows the membrane bending energy of equilibrium shapes for various normalized values of the difference of monolayer areas $\Delta a_{0}$ at the relative volume $v_{0}=0.95$. (Note that the same normalization procedure was used in both approaches, i.e. in the Euler method as well as in the third order method.) Each point of the curves of figure 1 corresponds to a shape of extremal bending energy determined by the Euler method. Obviously, all these shapes can be assigned to various classes. Shapes are considered to belong to the same class if they can be transformed into each other by continuous transitions through equilibrium shapes, and if they are characterized by the same symmetry properties. Five different classes of shapes are shown in figure 1 where they are denoted by Roman numbers.

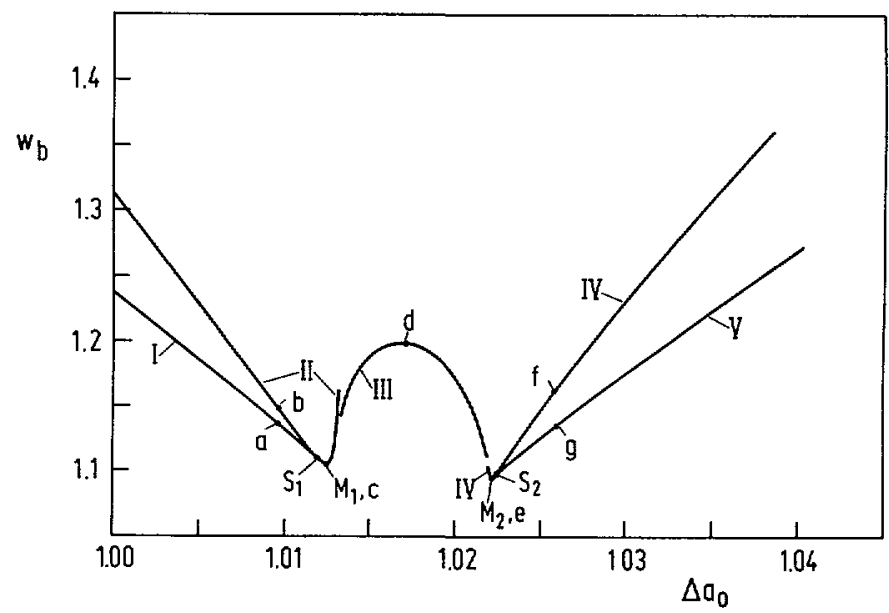

Fig. 1. - Relative membrane bending energies $w_{\mathrm{b}}$ of various axisymmetric equilibrium shapes calculated by the Euler method as functions of the relative leaflet area difference $\Delta a_{0}$ for $v_{0}=0.95$. I-V : different classes of shapes. $M_{1}$ and $M_{2}$ : minima of $w_{b}$ belonging to the classes II and IV, respectively, of mirror symmetric shapes. $S_{1}$ and $S_{2}$ : symmetry breaking points. Points a-g correspond to the shapes shown in figure $2 \mathrm{~A}$. 
Shapes of the oblate class II as well as of the prolate class IV are characterized by a mirror symmetry with respect to their equatorial plane (cf. Fig. $2 \mathrm{~A} ; \mathrm{b}, \mathrm{c}$, e and $\mathrm{f}$ ). As shown in figure 1 these two classes contain the shapes for which, at the given volume, the energy $w_{\mathrm{b}}\left(\Delta a_{0}\right)$ attains one of the local minima denoted by $\mathrm{M}_{1}$ and $\mathrm{M}_{2}$, respectively. At the points $S_{1}$ and $S_{2}$ of the classes II and IV new branches appear which correspond to non-mirror symmetric shapes belonging to the classes I and V, respectively (cf. Fig. 2A, a and g). Accordingly, $S_{1}$ and $S_{2}$ are called symmetry breaking points. Obviously, at the same $\Delta a_{0}$-values the shapes of the classes I and $\mathrm{V}$ have lower energies than the corresponding shapes of the classes II and IV, respectively. Shapes of classes I and II as well as of classes IV and $\mathrm{V}$ are continuously transformed into each other passing the symmetry breaking points $S_{1}$ and $S_{2}$, respectively. The shapes of the intermediate class III are non-mirror symmetric (cf. Fig. 2A, d). They involve on the left-hand side of the corresponding curve (Fig. 1) the characteristics of oblate shapes and on the right-hand side the characteristics of prolate ones. The behaviour of the $w_{\mathrm{b}}\left(\Delta a_{0}\right)$-curve of class III at its ends is still unclear because of numerical difficulties.

A

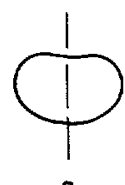

a

$\mathrm{B}$

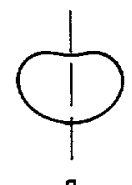

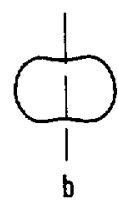

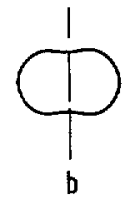

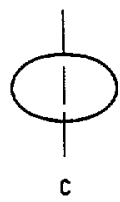

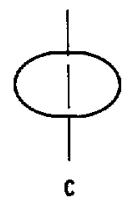

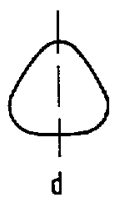

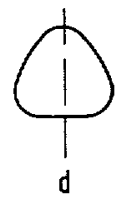

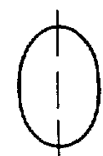

e

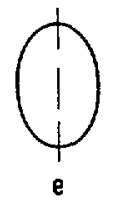

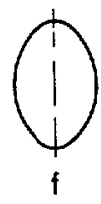

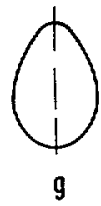

g
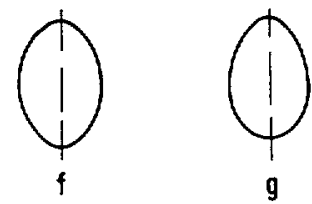

Fig. 2. - Examples of axisymmetric equilibrium shapes calculated by the Euler method (A) and the related shapes obtained by the third order method $(B)$ for points a-g in figures 1 and 3 , respectively. The shapes $b$ and $f$ of $(B)$ have been shown by the third order method to be unstable.

Let us compare the results of the Euler method with those of the third order method where in the computations the expansion in spherical harmonics is cut at $\ell_{\max }=10$. For that the results of the latter method are depicted in figure 3 using the analogous $w_{\mathrm{b}}\left(\Delta a_{0}\right)$-plot as in figure 1. The values of amplitudes as well as of Lagrange multipliers of several solutions belonging to different classes in figure 3 are listed in table $I$. The corresponding shapes and the related shapes obtained by the Euler method are shown in figures $2 \mathrm{~B}$ and $2 \mathrm{~A}$, respectively.

Obviously, the results obtained by the two methods are almost identical. The comparison of figures 1 and 3 shows that at the relative volume $v_{0}=0.95$ these two approaches yield principally the same $w_{\mathrm{b}}\left(\Delta a_{0}\right)$-dependence of an axisymmetric vesicle. Both approaches classify the calculated shapes in the same way, and the corresponding classes of shapes have the same symmetry properties. The mirror symmetry of the shapes of the classes II and IV (cf. Fig. 2B; $b, c$, e and $f$ ) is reflected in the zero values of the coefficients $X_{\ell}$ with odd numbers of $\ell$ (Tab. I). In addition, for these shapes the Lagrange multiplier $\lambda_{z}$ is zero since the requirement that the mass centre of the vesicle is the origin of the coordinate system is identically fulfilled. 


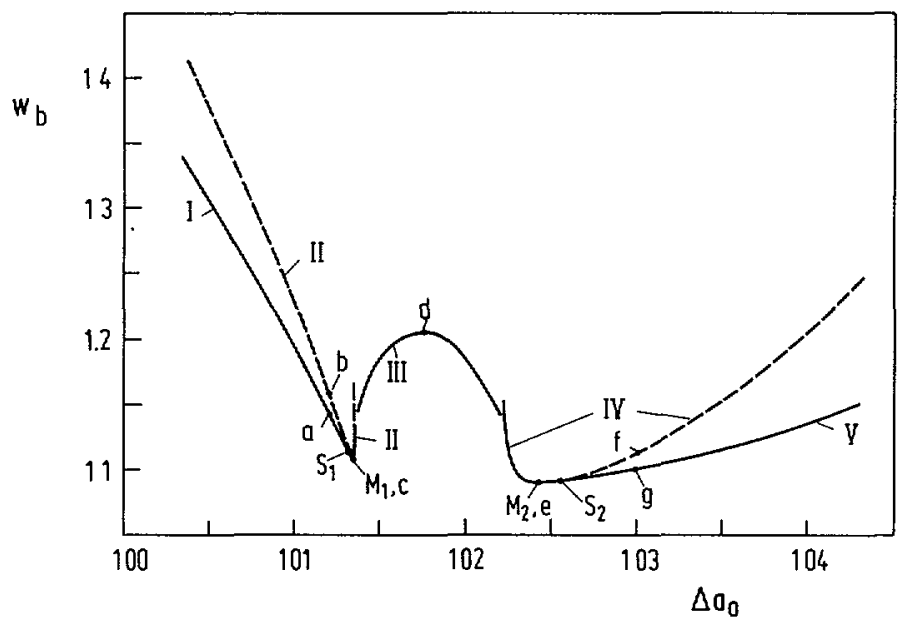

Fig. 3. - Relative membrane bending energies $w_{\mathrm{b}}$ of axisymmetric equilibrium shapes calculated by the third order method $\left(\ell_{\max }=10\right)$ as functions of the relative leaflet area difference $\Delta a_{0}$ for $v_{0}=0.95$. Broken lines indicate unstable shapes. Points a-g correspond to the shapes shown in figure $2 \mathrm{~B}$. For all other notations cf. figure 1.

Table I. - Relative differences of monolayer areas $\Delta a_{0}$, relative bending energies $w_{\mathrm{b}}$, amplitudes $X_{\mathfrak{l}}$ and Lagrange multipliers for the solutions corresponding to points a-g in figure 3. The corresponding shapes are shown in figure $2 \mathrm{~B}$.

\begin{tabular}{|l|r|r|r|r|r|r|r|}
\hline & \multicolumn{1}{c|}{$\mathrm{a}$} & $\mathrm{b}$ & $\mathrm{c}$ & $\mathrm{d}$ & $\mathrm{e}$ & \multicolumn{1}{c|}{$\mathrm{f}$} & $\mathrm{g}$ \\
\hline$\Delta a_{0}$ & 1.0120 & 1.0120 & 1.0135 & 1.0176 & 1.0244 & 1.0300 & 1.0300 \\
\hline$w_{\mathrm{b}}$ & 1.1427 & 1.1581 & 1.1057 & 1.2052 & 1.0892 & 1.1141 & 1.1004 \\
\hline$X_{0}$ & -0.0141 & -0.0147 & -0.0160 & -0.0069 & -0.0174 & -0.0140 & -0.0146 \\
$X_{1}$ & -0.0204 & 0.0000 & 0.0000 & 0.0037 & 0.0000 & 0.0000 & -0.0157 \\
$X_{2}$ & -0.2565 & -0.2720 & -0.2825 & -0.0441 & 0.2844 & 0.2452 & 0.2483 \\
$X_{3}$ & -0.0898 & 0.0000 & 0.0000 & 0.2111 & 0.0000 & 0.0000 & 0.0668 \\
$X_{4}$ & -0.0137 & -0.0499 & 0.0280 & 0.0223 & 0.0460 & 0.0870 & 0.0617 \\
$X_{5}$ & 0.0024 & 0.0000 & 0.0000 & -0.0024 & 0.0000 & 0.0000 & 0.0322 \\
$X_{6}$ & 0.0017 & -0.0041 & -0.0045 & 0.0151 & 0.0121 & 0.0397 & 0.0248 \\
$X_{7}$ & 0.0002 & 0.0000 & 0.0000 & 0.0043 & 0.0000 & 0.0000 & 0.0153 \\
$X_{8}$ & -0.0002 & -0.0002 & 0.0009 & 0.0001 & 0.0038 & 0.0187 & 0.0111 \\
$X_{9}$ & -0.0001 & 0.0000 & 0.0000 & 0.0017 & 0.0000 & 0.0000 & 0.0065 \\
$X_{10}$ & 0.0000 & 0.0000 & -0.0002 & 0.0008 & 0.0011 & 0.0080 & 0.0047 \\
\hline$\lambda_{a}$ & -24.3902 & -33.9840 & -3.1097 & -5.9977 & -2.1675 & 8.8526 & 2.3619 \\
$\lambda_{v}$ & 8.3268 & 11.0796 & 2.1846 & 4.2186 & 1.5623 & -3.6600 & -0.6300 \\
$\lambda_{\Delta}$ & 24.9017 & 36.1541 & 0.0000 & 0.0000 & 0.0000 & -6.5357 & -2.4747 \\
$\lambda_{z}$ & 0.2706 & 0.0000 & 0.0000 & 0.0310 & 0.0000 & 0.0000 & 0.2673 \\
\hline
\end{tabular}


The points c, d and e (Fig. 3) are located at extrema of the $w_{\mathrm{b}}\left(\Delta a_{0}\right)$-curves of classes II, III and IV, respectively. Therefore, the Lagrange multiplier $\lambda_{\Delta}$ vanishes for the corresponding solutions (Tab. I ; c, d and e). In general, all shapes of the different classes are characterized by $X_{2}$ as the amplitude with the greatest absolute value. The only exception is the middle region of the class of intermediate shapes (III) where $X_{3}$ dominates.

The calculated $X_{\ell}$-values may be used for a rough evaluation of the influence of the limited number of spherical harmonics on the results of the method. If for higher $\ell$ the values of $\left|X_{\ell}\right|$ become very small it may be expected that the error resulting from the use of a finite number of spherical harmonics is sufficiently small. This is in fact the case for most points of the curves in figure 3. (Moreover, calculations performed with $\ell_{\max }=6$ show that a moderate reduction of the $\ell_{\max }$-value has only a minor influence on the results.) On the other hand, high values of $\left|X_{\ell}\right|$ at higher $\ell$ indicate that in this case the use of spherical harmonics meets with difficulties.

For a given solution the accuracy of the third order method can be evaluated as follows. On the one hand, the amplitudes $X_{\ell}$ of this solution have been calculated by the third order method for input values $a_{0}=1, v_{0}$ and $\Delta a_{0}$ in such a way that they fulfil equations (21-23). On the other hand, the initial equations (6-9) do not involve the approximations of the third order method. Thus, if the function $r=r(\vartheta)=r_{0}+\Sigma X_{\ell} P_{\ell}(\cos \vartheta)\left(\ell=0 \ldots \ell_{\max }\right)$ with the same coefficients $X_{\ell}$ is used in the axisymmetric versions of these initial equations, and if the values of the constraints are recalculated by a numerical integration of the resulting expressions (e.g. by Simpsons rule) these values will, in general, be different from the input values, and the corresponding differences will globally represent the effect of the approximations of the third order method. The latter values of the constraints are denoted by $a_{\text {int }}, v_{\text {int }}$ and $\Delta a_{\text {int }}$, respectively. It is worth mentioning that the values of $v_{0}$ and $v_{\text {int }}$ must be the same since no terms have been neglected to obtain equation (15). On the other hand, the differences between the input values $a_{0}=1$ and $\Delta a_{0}$ and the values $a_{\text {int }}$ and $\Delta a_{\text {int }}$, respectively, can be used for each solution as a criterion of the accuracy of the method. In addition to that, one may calculate the difference $w_{\mathrm{b}}-w_{\text {int }}$ by the same procedure. The resulting relative differences were calculated as functions of the volume $v_{0}$ for those two characteristic points of the $w_{\mathrm{b}}=w_{\mathrm{b}}\left(\Delta a_{0}\right)$-curve where $w_{\mathrm{b}}$ attains a local minimum (cf. points $\mathrm{M}_{1}$ and $\mathrm{M}_{2}$ in Fig. 3 ). The results are given in figures $4 \mathrm{a}$ and $4 \mathrm{~b}$, respectively. As expected, the accuracy of the third order method decreases with decreasing values of $v_{0}$. For $v_{0}=0.95$ the degree of inconsistency of the model is of the order $10^{-3}$ to $10^{-2}$. Comparing figures $4 \mathrm{a}$ and $4 \mathrm{~b}$ it can be seen that at the first local minimum of $w_{\mathrm{b}}\left(\Delta a_{0}\right)$ characterized by low $\Delta a_{0}$-values the accuracy of the third order method is higher than at the second local minimum characterized by higher $\Delta a_{0}$-values.

The third order method should be regarded as an improvement of the analogous method which involves only the terms up to the second order in the deviation from a sphere. Neglecting also the cubic terms in equations (14-16) it can easily be proved that these three constraints are not independent anymore. Within the second order approximation the following equation holds at equilibrium :

$$
r_{0}^{3}-3 r_{0}^{2} \Delta a_{0}+3 r_{0}-v_{0}=0
$$

Since $r_{0}$ is fixed by the relation $r_{0}=\left(v_{0}\right)^{1 / 3}$ it follows that for a given relative volume $v_{0}$ all classes are condensed into one point characterized by the relative difference of monolayer areas $\Delta a_{0}=\left(v_{0}\right)^{-1 / 3}$ For $v_{0}=0.95$ this point is at $\Delta a_{0} \approx 1.0172$. It follows that this second order approximation can not reproduce the results of the Euler method presented in figure 1. There are two other reasons for taking into account at least the cubic terms of the deviation from a sphere in the Taylor expansion. First, within the second order approximation 


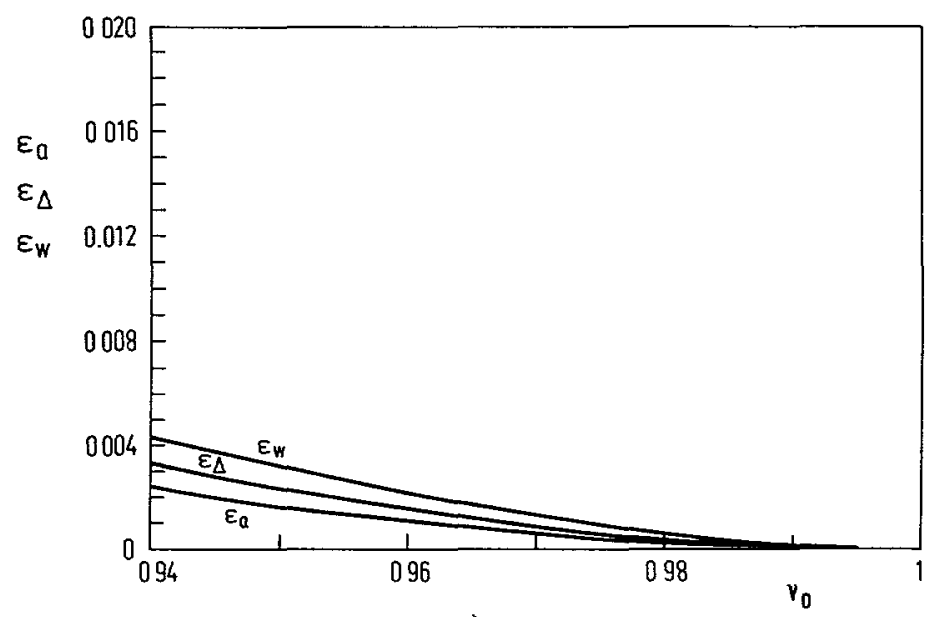

a)

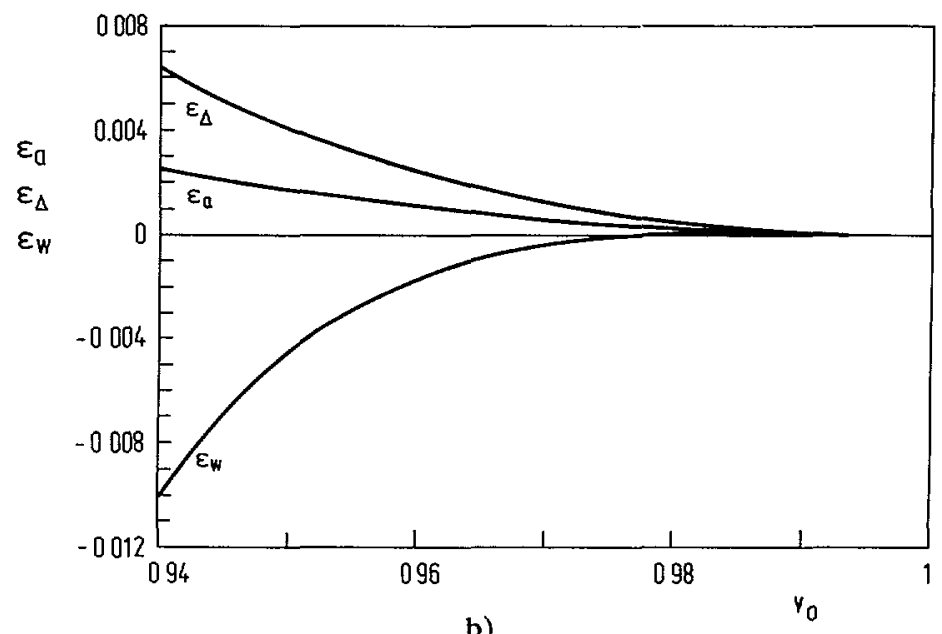

b)

Fig. 4. - Relative differences $\varepsilon_{a}, \varepsilon_{\Delta}$ and $\varepsilon_{w}$ between the values of model quantities of the third order method and the integrated values calculated by the exact equations (6), (7) and (9) as functions of the relative volume $v_{0}: \varepsilon_{a}=\left(a_{0}-a_{\mathrm{nt}}\right) / a_{0}, \varepsilon_{\Delta}=\left(\Delta a_{0}-\Delta a_{\mathrm{int}}\right) / \Delta a_{0}$ and $\varepsilon_{w}=\left(w_{\mathrm{b}}-w_{\mathrm{mt}}\right) / w_{\mathrm{b}}$. a) For the $w_{\mathrm{b}}$-minimum of class II (cf. $\mathrm{M}_{1}$ in Fig. 3). b) For the $w_{\mathrm{b}}$-minimum of class IV (cf. $\mathbf{M}_{2}$ in Fig. 3 ).

one gets for axisymmetric shapes $U_{\ell}^{(2)}=\left(U_{\ell_{0}}\right)^{2}$. Consequently, this approximation does not distinguish between positive and negative values of $U_{\mathfrak{\ell}_{0}}$. Different signs of $U_{\mathfrak{f}_{0}}$, however, may correspond to completely different shapes [3, 17, 18]. Second, within the second order the bending energy and the constraints are degenerated with respect to the coefficients of those spherical harmonics which characterize the nonaxisymmetric features of the solutions. i.e. they depend only on the sums $U_{q}^{(2)}$ but not on the amplitudes $U_{P_{m}}$ (cf. Eqs. (20-24). Thus, the third order approximation is the lowest order in the Taylor expansion which enables also the determination of nonaxisymmetric shapes of phospholipid vesicles.

Using the third order method the stability of the resulting shapes can be determined by the relatively simple procedure described in section 5 . A solution is stable if all eigenvalues of the 
matrix of second derivatives of the bending energy with respect to independent amplitudes of spherical harmonics are positive. As the result of this stability analysis within the axisymmetric case it has been shown that the mirror symmetric shapes of both classes II and IV become unstable at the symmetry breaking points $S_{1}$ and $S_{2}$, respectively.

Accordingly, curves in figure 3 which correspond to unstable shapes are drawn as broken lines. One may conclude that these unstable mirror symmetric shapes actually cannot be observed. For the corresponding $\Delta a_{0}$-values the vesicle can assume only the non-mirror symmetric shapes belonging to the classes I and $\mathrm{V}$.

\section{Discussion of the axisymmetric case.}

As shown in the previous section the third order method and the Euler method yield qualitatively the same results. On the other hand, there are considerable differences between the values of the model quantities obtained by the two methods. In the following, the origins of these differences are studied in more detail.

The third order method involves approximations in connection with the Taylor expansion in the deviation from a sphere as well as with the use of a finite number of spherical harmonics within the Ritz procedure. Although the proof of the convergence of the Ritz procedure is problematic for the given variational problem it can be expected that for sufficiently high values of $\ell_{\max }$ the accuracy of the third order method is mainly limited by the third order expansion. This statement can be proved by another way of calculating axisymmetric vesicle shapes which is called here the modified Euler method. By this method shapes are determined on the basis of the third order expansion in the displacement from a sphere but without expansion in spherical harmonics which allows to estimate the effects of these two different approximations. The basic idea of the modified Euler method is to apply an Euler-Lagrange ansatz to the axisymmetric (third order) versions of equations (13-16) and (31). Partial derivatives with respect to the polar angle $\varphi$ must vanish, so that the resulting Lagrange function can be used in the corresponding Euler equation with the azimuthal angle $\vartheta$ being the only independent variable. The resulting system of differential equations is solved in an analogous way as within the Euler method (cf. [6, 8]), i.e. without further approximations. Note that the primary purpose of this modified Euler method is not the determination of equilibrium shapes but just the evaluation of the error resulting from the use of a third order Taylor expansion.

Figure 5 shows the results of the modified Euler method under the same conditions as used to obtain figures 1 and 3 . The lines belonging to classes $I I$ and $V$ in figure 5 could not be prolonged in direction of higher values of $\Delta a_{0}$ because of numerical difficulties. As expected the modified Euler method yields globally the same results as the Euler method (Fig. 1) and the third order method (Fig. 3).

The influence of the use of a finite number of spherical harmonics on the accuracy of the solutions of the third order method can be estimated by comparing the results of this method (for $\ell_{\max }=10$, Fig. 3) with those of the modified Euler method (Fig. 5). For lower $\Delta a_{0}$-values $\left(\Delta a_{0} \leqslant 1.025\right)$ the results of these two methods are almost identical. This is in accordance with the above-mentioned minor effect of amplitudes $X_{\ell}$ at higher $\ell$ due to their small absolute values for almost all solutions shown in figure 3 . This effect can also be proved by calculating vesicle shapes for varying values of $\ell_{\max }$ and comparing the results with those of the modified Euler method. Such calculations are performed for $\ell_{\max }=5$ to 15 at the two $\Delta a_{0}$-values corresponding to points $a$ and $g$ in figure 3 , respectively. The relative differences between the bending energies $w_{\mathrm{b}}^{(\ell)}$ obtained in this way and the corresponding values $w_{\mathrm{b}}^{(u)}$ calculated by the modified Euler method are depicted in figures $6 \mathrm{a}$ and $6 \mathrm{~b}$, respectively. It can be seen that with increasing $\ell_{\max }$-values the results of the third order method approach 


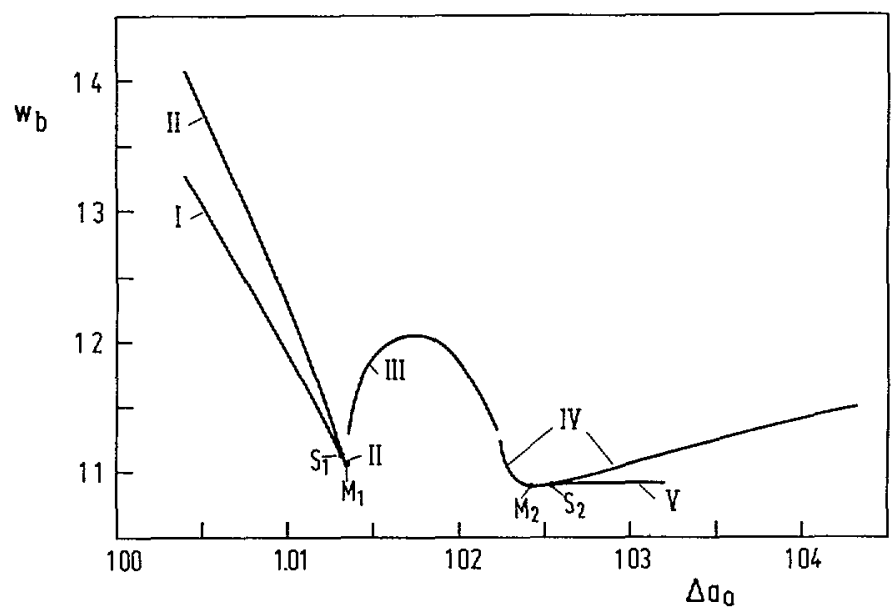

Fig. 5. - Relative membrane bending energies $w_{\mathbf{b}}$ of various axisymmetric equilibrium shapes calculated by the modified Euler method as functions of the relative leaflet area difference $\Delta a_{0}$ for $v_{0}=0.95$. For notations $\mathrm{cf}$. figure 1 .

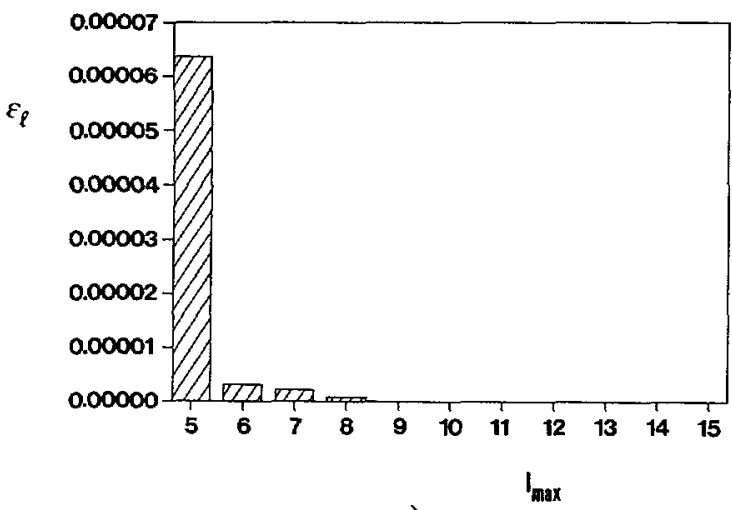

a)

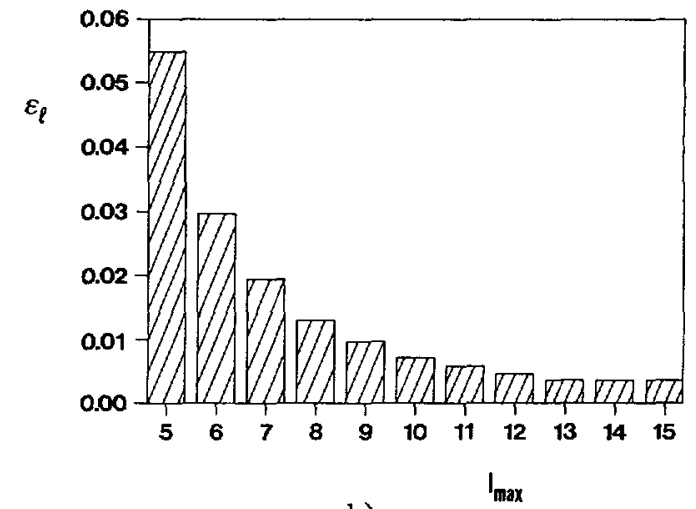

b)

Fig. 6. - Relative differences $\varepsilon_{\ell}$ between the bending energies $w_{b}^{(\ell)}$ calculated for various values of $\ell_{\max }$ by the third order method and the corresponding value $w_{\mathrm{b}}^{(u)}$ obtained by the modified Euler method in dependence on $\ell_{\max } \cdot \varepsilon_{\ell}=\left(w_{\mathrm{b}}^{(\ell)}-w_{\mathrm{b}}^{(u)}\right) / w_{\mathrm{b}}^{(u)}$. a) For $\Delta a_{0}=1.012$ (cf. point a in Fig. 3). b) For $\Delta a_{0}=1.03$ (cf. point $\mathrm{g}$ in Fig. 3).

those of the modified Euler method. The differences between the results of these two methods are already for $\ell_{\max }=6$ smaller than the differences between the results of the modified Euler method and the Euler method. The latter differences represent the error which is caused by the third order expansion. It is evident, therefore, that the increase of $\ell_{\max }$ up to very high values is not necessary since it would not improve the results of the third order method.

It can be concluded that the main error of the third order method is caused by the third order Taylor approximation. This error can be estimated by comparing the results of the Euler method (Fig. 1) and the modified Euler method (Fig. 5). The differences between these two methods become considerable especially for higher values of the relative leaflet area difference $\Delta a_{0}$ (cf. classes IV and V in Figs. 1 and 5). Obviously, the term « nearly spherical 
shape » does not comprise simply all shapes of a volume $v_{0}$ close enough to $v_{0}=1$ but for every given volume only those shapes whose $\Delta a_{0}$-values are within a certain limited region. The differences in the $w_{\mathrm{b}}\left(\Delta a_{0}\right)$-curves shown in figures 1 and 5 may be explained by the fact that for the shapes belonging to the classes IV and $\mathrm{V}$ the deviation $u$ becomes quite large at the poles of these shapes $\left(|u| \approx r_{0} / 2\right.$, cf. Fig. 2 , $f$ and $g$ ) so that the requirement that the shapes must be nearly spherical is hardly fulfilled. It follows, that in general the accuracy of the third order method depends on the degree to which the calculated shape is nearly spherical.

Within the Euler method all calculations are independent of the origin of the chosen coordinate system (cf. [6]). It is evident that this independence is lost within the methods using a third order approximation. In these methods the model quantities are expressed in terms of the deviation from a corresponding sphere whose centre is the origin of the coordinate system. Consequently, these deviations are different for different positions of the reference frame. In particular, the contributions of neglected terms of higher orders in $u$ are changing for varying positions of the corresponding sphere, so that the variational procedure yields new values of the model quantities as well as new shapes. In figure 7 the bending energy determined by the modified Euler method for a vesicle shape of class I $\left(v_{0}=0.95, \Delta a_{0}=1.013\right)$ is shown as a function of $z_{m}$, i.e. of the distance between the mass centre of the vesicle and the origin of the coordinate system. The dot at $z_{m}=0$ corresponds to the case where origin and mass centre are identical, i.e. the point used above to fix the coordinate system in the third order method as well as in the modified Euler method. If the coordinate system were not fixed the minimization of the bending energy would be performed also with respect to $z_{m}$, i.e. along the curve shown in figure 7. Obviously, for increasing values of $z_{m}$ the bending energy would decrease. However, a large shift of the corresponding sphere relative to a given shape increases the average absolute deviation of this shape from the corresponding sphere and, therefore, also the error of the third order expansion. In fact, the error of the method becomes with increasing $z_{m}$-values so large that the corresponding results must be considered to have no meaning within the present model. This behaviour was also tested for the relative volume $v_{0}=0.999$ and it was observed that an artificial dependence of the bending energy on $z_{m}$ can also be found for almost spherical vesicle shapes.

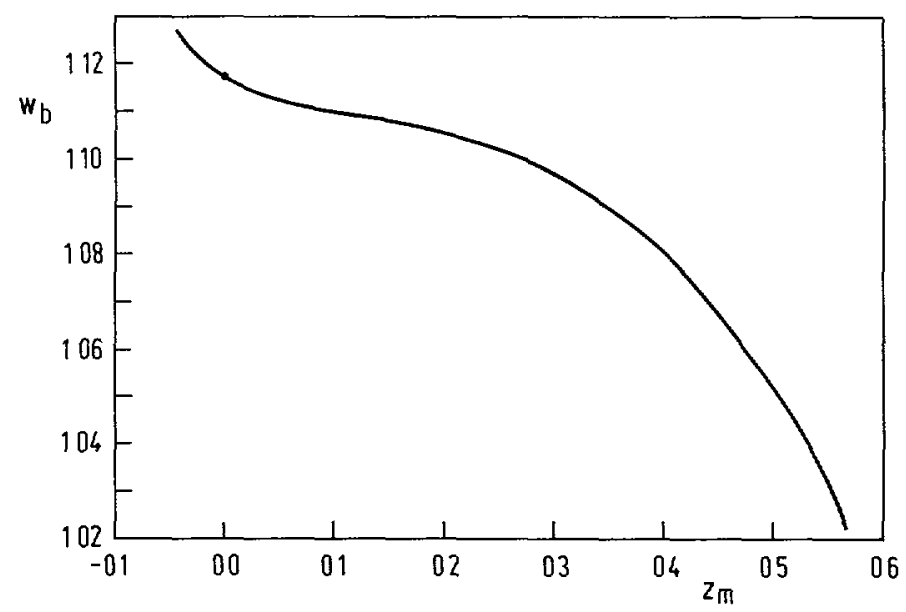

Fig. 7. - Bending energy $w_{b}$ of axisymmetric shapes calculated by the modified Euler method as function of the distance between the origin of the coordinate system and the mass centre of the vesicle. Parameters : $v_{0}=0.95, \Delta a_{0}=1.013$. 
It follows that leaving the origin of the coordinate system free would cause a completely wrong behaviour of the model. In this work the mass centre of the vesicle was chosen as the centre of the corresponding sphere, i.e. the origin of the coordinate system. Of course, there are also other possibilities for choosing the position of the reference frame. For example, one could require that the average absolute deviation of the vesicle shape from the corresponding sphere attains a minimum. The present choice was made mostly for practical reasons.

Concerning the stability of the calculated vesicle shapes the third order method represents an improvement compared with the Euler method since it enables the stability analysis by a relatively simple procedure. It is important to note that the results of the stability analysis performed within the third order method should be carefully applied for a generalization. Results concerning the stability of shapes are valid only within the frame given by the finite number of spherical harmonics used. In other words, the stability analysis of a given solution is as general as the method used to calculate the corresponding shape. Taking into account the good correspondence of the results of the Euler method and the third order method one may expect, however, that the results of the stability analysis are also valid for nearly spherical vesicle shapes calculated by the Euler method.

Summarizing the results of the application of the third order method to axisymmetric shapes one may conclude that this method reproduces the main features of the results obtained before by the Euler method. It is suggested, therefore, that the approach presented in this paper is applicable to the general case of the determination of nearly spherical vesicle shapes without symmetry restrictions.

\section{Application of the third order method without symmetry restrictions.}

All mathematical details needed to determine equilibrium shapes without restricting the calculations to a certain symmetry are given in sections $2-4$. The corresponding computations are performed with $\ell_{\max }=6$ at the relative volume $v_{0}=0.95$. That means that for every shape the values of 49 amplitudes together with those of 9 Lagrange multipliers are determined solving the corresponding system of nonlinear equations. As the main result of this procedure the bending energies $w_{\mathrm{b}}$ of equilibrium shapes are again calculated for varying values of the difference of monolayer areas $\Delta a_{0}$. The results are shown in figure 8 . (Note that the scales in this figure are different from those used in Figs. 1, 3 and 5.)

It can be seen that all axisymmetric solutions are also obtained within the general treatment. For these solutions the computation yields $X_{\ell_{m}}^{r}=0$ and $X_{\ell_{m}}^{l}=0$ if $m \neq 0$ (for the definition of these amplitudes cf. Eq. (39)). In figure 8 the same notation as in figure 3 was used in order to identify the characteristics of the five classes of axisymmetric shapes.

The only new class obtained by the general treatment is denoted by VI. Shapes belonging to this class are not axisymmetric. Nevertheless they are characterized by a high symmetry, namely by a threefold mirror symmetry where every symmetry plane contains a pair of axes of the Cartesian coordinate system. Figure 9 shows the cross-sections of a nonaxisymmetric shape of class VI $\left(\Delta a_{0}=1.02\right)$ with its three symmetry planes. For the shapes of class VI the only non-zero amplitudes are those amplitudes $X_{\ell_{m}}^{r}$ for which both $\ell$ and $m$ are even numbers. The values of non-zero amplitudes as well as of Lagrange multipliers calculated for the shape shown in figure 9 are given in table II. It is clear that for shapes of such a symmetry all six Lagrange multipliers which correspond to the constraints fixing the coordinate system are zero.

The nonaxisymmetric vesicle shapes of class VI can be continuously transformed into the axisymmetric ones of the classes II and IV by passing the symmetry breaking points $S_{3}$ and $S_{4}$, respectively. $S_{3}$ and $S_{4}$ are not identical with $M_{1}$ and $M_{2}$ but are located at lower 


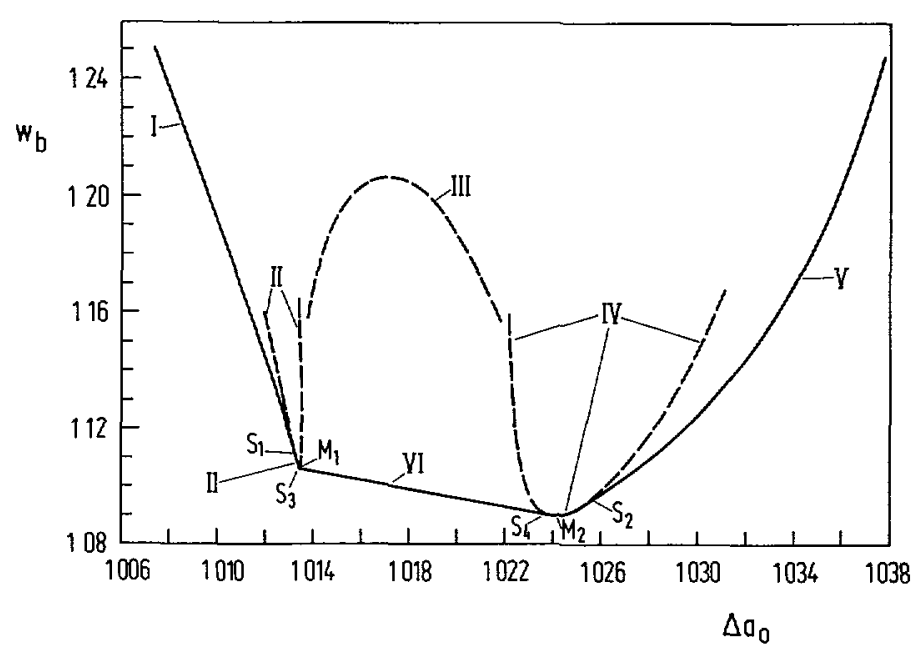

Fig. 8. - Relative membrane bending energies $w_{\mathrm{b}}$ of equilibrium shapes of different symmetries calculated by the general third order method $\left(\ell_{\max }=6\right)$ as functions of the relative leaflet area difference $\Delta a_{0}$ for $v_{0}=0.95$. Broken lines indicate unstable shapes. I-V : different classes of axisymmetric shapes. VI : class of nonaxisymmetric shapes. $M_{1}$ and $M_{2}$ : minima of the $w_{b}$-curves of classes II and IV, respectively. $S_{1}-S_{4}$ : symmetry breaking points.
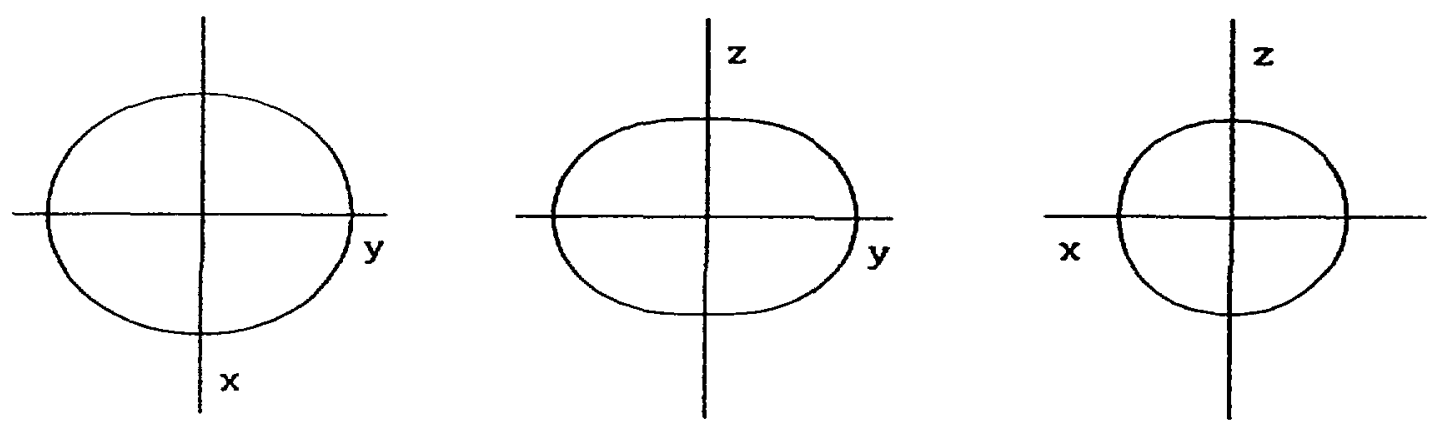

Fig. 9. - Cross-sections of a nonaxisymmetric shape obtained by the general third order method $\left(\ell_{\max }=6, v_{0}=0.95, \Delta a_{0}=1.02\right)$ with its three symmetry planes (cf. Fig. 10, c).

$\Delta a_{0}$-values, respectively. The transformation of an oblate shape (class II) into a prolate one (class IV) can easily be understood as a transformation along the curve of class VI in the direction of increasing $\Delta a_{0}$-values. During this transformation the symmetry breaking points $S_{3}$ and $S_{4}$ are passed in this order. Let us start such a transformation from an oblate shape whose symmetry axis is the $z$-axis of the Cartesian coordinate system. Beyond the point $\mathrm{S}_{3}$ this shape is more and more laterally elongated in direction of the $x$ - or the $y$-axis. At $\mathrm{S}_{4}$ the resulting axisymmetric prolate shape has one of these two axes as symmetry axis. If it is then rotated by $\Delta \vartheta=\pi / 2$ in such a way that the $z$-axis is again the symmetry axis, this prolate shape is identical with the corresponding shape obtained within the axisymmetric case.

It shall be noted that this result concerning the existence of nonaxisymmetric shapes is in accord with [20] where nonaxisymmetric ellipsoids with three reflection planes were supposed to fill the gap between oblate and prolate shapes in the phase diagram. 
Table II. - Values of model quantities, amplitudes and Lagrange multipliers characterizing a nonaxisymmetric shape obtained by the general third order method. The shape is shown in figure 9.

\begin{tabular}{|c|c|c|}
\hline parameters : & $\begin{array}{r}v_{0}= \\
\Delta a_{0}=\end{array}$ & $\begin{array}{l}0.95000 \\
1.02000\end{array}$ \\
\hline bending energy: & $w_{\mathrm{b}}=$ & 1.09602 \\
\hline non-zero amplitudes: & $\begin{array}{l}X_{00}^{r}= \\
X_{20}^{r}= \\
X_{22}^{r}= \\
X_{40}^{r}= \\
X_{42}^{r}= \\
X_{44}^{r}= \\
X_{60}^{r}= \\
X_{62}^{r}= \\
X_{64}^{r}= \\
X_{66}^{r}=\end{array}$ & $\begin{array}{r}-0.01708 \\
-0.23194 \\
0.11814 \\
0.02279 \\
-0.01554 \\
0.00989 \\
-0.00373 \\
0.00295 \\
-0.00194 \\
0.00137\end{array}$ \\
\hline Lagrange multipliers : & \multicolumn{2}{|c|}{$\begin{aligned} \lambda_{a} & =-4.37637 \\
\lambda_{v} & =2.54524 \\
\lambda_{\Delta} & =1.51796 \\
\lambda_{\mathrm{s}_{3}} & =0.00000\end{aligned}$} \\
\hline \multicolumn{3}{|c|}{ stability : all signs of the 40 eigenvalues are positiv } \\
\hline
\end{tabular}

The stability analysis performed within the general case yields in the neighbourhoods of the symmetry breaking points $S_{1}$ and $S_{2}$ the same results as within the axisymmetric case. In addition, it is shown that all shapes of class III are unstable with respect to nonaxisymmetric deformations. Furthermore, the shapes of the classes II and IV become unstable at $S_{3}$ (with increasing $\Delta a_{0}$ ) and $S_{4}$ (with decreasing $\Delta a_{0}$ ), respectively. In figure 8 all curves belonging to unstable solutions are drawn as broken lines. It is seen that only one shape is stable for every $\Delta a_{0}$-value. In all cases the stable shape is characterized by the lowest energy $w_{\mathrm{b}}$ at the given $\Delta a_{0}$.

In this way the minimum $\mathrm{M}_{2}$ (class IV) of the $w_{\mathrm{b}}\left(\Delta a_{0}\right)$-curve is found to be the global minimum of this dependence. The corresponding prolate shape (Fig. $2 \mathrm{~B}$, e for $\ell_{\max }=10$ ) can be interpreted as the most « relaxed » shape at the given relative volume $v_{0}=0.95$. That means that the vesicle would assume this shape if $\Delta a_{0}$ were allowed to change by some slow process, for example, by a transversal movement of phospholipid molecules from one monolayer to the other (so-called flip-flop) under quasi-equilibrium conditions.

Summarizing the results of the third order method in the general case one can say that this method yields a complete picture of the behaviour of the shape of a nearly spherical vesicle. It is possible to describe the shape transformations of such a vesicle with respect to varying differences between the areas of the two monolayers $\left(\Delta a_{0}\right)$. There are no gaps left between different stable equilibrium shapes in the $w_{\mathrm{b}}\left(\Delta a_{0}\right)$-diagram, i.e. a given stable shape can be 
transformed into any other stable shape in a continuous manner by changing $\Delta a_{0}$. In this way a vesicle can attain shapes which are characterized by the following symmetry elements :

(i) one symmetry axis (classes I and V);

(ii) one symmetry axis and one equatorial symmetry plane (classes II and IV);

(iii) three symmetry planes (class VI).

Figure 10 shows a series of stable equilibrium shapes belonging to different classes and, therefore, characterized by different symmetries. These shapes were obtained by the use of the general third order method for various values of $\Delta a_{0}\left(v_{0}=0.95, \ell_{\max }=6\right)$.

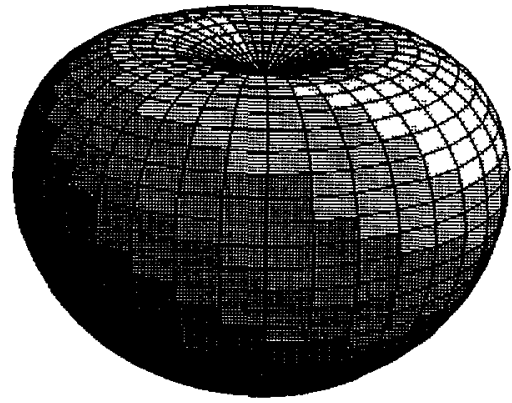

a)

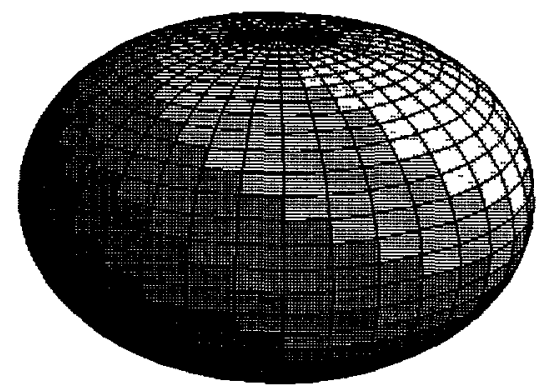

b)

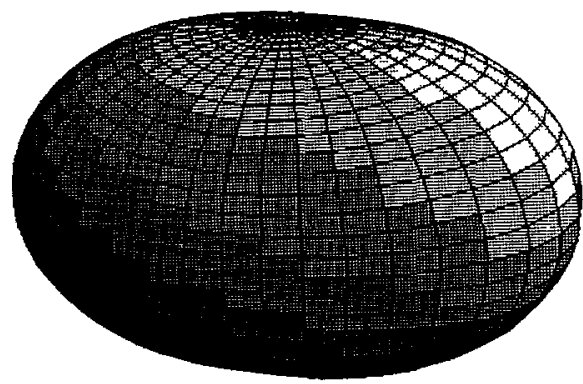

c)

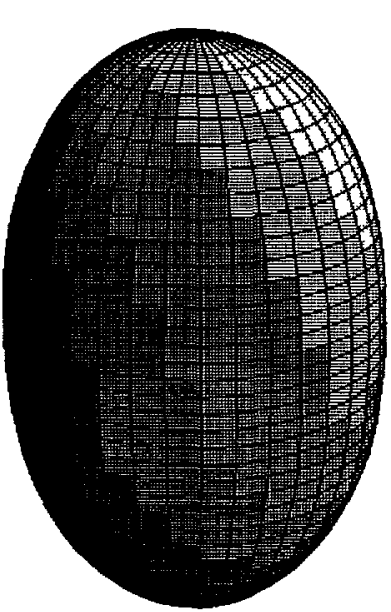

d)

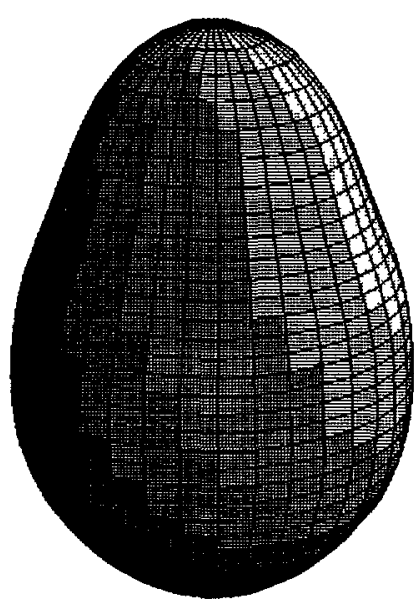

e)

Fig. 10. - Examples of stable equilibrium shapes of different classes calculated by the general third order method $\left(\ell_{\max }=6, v_{0}=0.95\right.$ ). a) $\Delta a_{0}=1.01$, class I; b) $\Delta a_{0}=1.0135$, class II ; c) $\Delta a_{0}=1.02$, class VI ; d) $\Delta a_{0}=1.0241$, class IV ; e) $\Delta a_{0}=1.03$, class $\mathrm{V}$. The shapes a, b, $\mathrm{d}$ and $\mathrm{e}$ are axisymmetric whereas shape $\mathrm{c}$ is nonaxisymmetric. The prolate shapes $\mathrm{d}$ and e were rotated by $\Delta \vartheta=\pi / 2$ with respect to those positions in which they are continuously obtained from shape $\mathrm{c}$ by increasing $\Delta a_{0}$. This was done in such a way that their symmetry axis has again a vertical direction. 


\section{Conclusions.}

In the present paper a mathematical model to determine nearly spherical equilibrium shapes of phospholipid vesicles and their stability is developed without restrictions to any symmetry. It is based on the bilayer couple concept and assumes that equilibrium shapes correspond to the minimum of the membrane bending energy at constant values of the membrane area, the vesicle volume and the difference of areas of the two leaflets of the phospholipid bilayer.

The bending energy as well as the three constraints are expanded up to the fourth order in terms of the deviation from a sphere. Taking into account all terms up to the third order the resulting integrals are calculated by expressing the deviation as a series of spherical harmonics. It was found that the coordinate system has to be fixed which was done by choosing the mass centre of the vesicle as its origin. Furthermore, two of its axes were fixed in order to prevent a rotation of the reference frame. This procedure to fix the coordinate system yielded six additional constraints. For the calculation of equilibrium shapes a Ritz procedure was applied by searching for those sets of amplitudes of spherical harmonics which minimize the expression for the bending energy and, at the same time, fulfil the nine constraints.

Furthermore, a procedure to determine the stability of shapes obtained by the third order method is developed. Hitherto, a complete stability analysis has not been performed within the Euler method. The stability analysis presented here takes into account the constraints by distinguishing between dependent and independent amplitudes of spherical harmonics. A vesicle shape is stable if all eigenvalues of the matrix of second derivatives of the bending energy with respect to independent amplitudes are positive. The resulting procedure was applied to the axisymmetric version of the third order method as well as to the method in its general form. Since the general third order method yields many different solutions the determination of their stability is very useful in order to decide whether the corresponding vesicle shapes can be assumed to exist.

For the special case of axisymmetric shapes the numerical results of the third order method were compared with those of the Euler method which does not involve the approximations of the former method. It was shown that up to a certain precision the third order method reproduces the results obtained by the Euler method. Since the main error of the third order method was found to be due to the Taylor expansion in terms of the deviation from a sphere it must be required that the shapes are nearly spherical.

Applying the general third order method, stable vesicle shapes of different classes were calculated. It was shown that at the relative volume $v_{0}=0.95$ the global minimum of the membrane bending energy with respect to varying $\Delta a_{0}$-values corresponds to a prolate axisymmetric shape which is also mirror symmetric with respect to its equatorial plane. Starting from this shape one passes at decreasing $\Delta a_{0}$ the region of nonaxisymmetric shapes and arrives eventually at an axi- but non-mirror symmetric cupped shape. In contrast to that, an increase of $\Delta a_{0}$ leads to an axisymmetric pear-like shape. Within the present model a further change of $\Delta a_{0}$ to even lower or higher values, respectively, is problematic since the corresponding shapes are not nearly spherical anymore. As shown by previous studies using the Euler method the limiting shape of class I (low $\Delta a_{0}$-values) is a sphere containing another invaginated sphere whose membrane is oriented inside out, i.e. this invaginated sphere encloses external medium. On the other hand, the limiting shape of class $V$ (high $\Delta a_{0}$-values) consists of two spheres sitting on top of each other. In this case both spheres contain the vesicle medium, i.e. the smaller sphere is evaginated $[6,9,10]$. Assuming that these limiting shapes are also stable they may be taken into consideration in order to supplement the description of the general behaviour of vesicle shapes with relative volumes close enough to unity. 
Concerning the Taylor expansion in terms of the deviation from a sphere it was shown that the second order of this expansion is not sufficient to calculate a $w_{\mathrm{b}}\left(\Delta a_{0}\right)$-dependence of axisymmetric shapes. Taking into account also the cubic terms a good agreement of the third order method and the Euler method was obtained for axisymmetric vesicles with relative volumes $v_{0} \geqslant 0.95$. Furthermore, it was found that the third order method can describe also nonaxisymmetric shapes which were assumed to exist in the gap of the phase diagram of axisymmetric shapes between oblate and prolate ellipsoids $[8,20]$. For nearly spherical vesicles these nonaxisymmetric shapes are ellipsoids with three reflection planes.

In section 2, the equations for the bending energy (13) and the three constraints (14-16) contain also the fourth order terms of the Taylor expansion. In principle, these terms could be included into the calculations, and the corresponding results would be expected to be more accurate than those of the third order method. However, taking into account the good agreement of nearly spherical shapes obtained by the third order method and the Euler method, respectively, it can not be expected that for these shapes the inclusion of fourth order terms would lead to qualitatively new results. On the other hand, if the deviation $u$ is not small compared with $r_{0}$ any Taylor expansion will meet with difficulties, so that for the calculation of the corresponding equilibrium shapes other mathematical methods have to be envisaged.

\section{Acknowledgements.}

We thank E. Farge and P. F. Devaux for sending a copy of their recent paper prior to publication.

\section{Appendix.}

\section{Calculation of integrals by using spherical harmonics.}

To obtain equations (20-25) from equations (13-16) one has to expand the displacement $u(\vartheta, \varphi)$ in spherical harmonics (cf. Eq. (17)) and to calculate the corresponding integrals. This can be done by using the properties of spherical harmonics, e.g. their orthonormality and the eigenvalue equation : $\Delta Y_{\ell_{m}}=-\ell(\ell+1) Y_{\ell_{m}}$. In addition, for the calculation of products of three spherical harmonics the coupling rule :

$$
Y_{\ell_{1} m_{1}} Y_{\ell_{2} m_{2}}=\sum_{\ell} A\left(\ell_{1} \ell_{2} \ell ; m_{1} m_{2}\right) Y_{\ell, m_{1}+m_{2}}
$$

can be used. The coefficients $A\left(\ell_{1} \ell_{2} \ell ; m_{1} m_{2}\right)$ are defined by the Clebsch-Gordan coefficients $C\left(\ell_{1} \ell_{2} \ell ; m_{1} m_{2}\right)$ (cf. [23]) as follows :

$$
A\left(\ell_{1} \ell_{2} \ell ; m_{1} m_{2}\right)=\sqrt{\frac{\left(2 \ell_{1}+1\right)\left(2 \ell_{2}+1\right)}{4 \pi(2 \ell+1)}} C\left(\ell_{1} \ell_{2} \ell ; m_{1} m_{2}\right) C\left(\ell_{1} \ell_{2} \ell ; 00\right) \text {. }
$$

Indices $\ell$ and $m_{i}(i=1,2)$ have to fulfil the conditions (26-28) where $\ell$ replaces $\ell_{3}$. The integral of products of three spherical harmonics can then be calculated in the following way

$$
\int Y_{\ell_{1} m_{1}} Y_{\ell_{2} m_{2}} Y_{\ell_{3} m_{3}}^{*} \mathrm{~d} \Omega=A\left(\ell_{1} \ell_{2} \ell_{3} ; m_{1} m_{2}\right) \delta_{m_{1}+m_{2}, m_{3}} .
$$


In a first step all expressions containing the $\nabla$-operator are transformed by the use of the relation

$$
\nabla u_{1} \cdot \nabla u_{2}=\frac{1}{2} \Delta\left(u_{1} u_{2}\right)-\frac{1}{2} u_{1} \Delta u_{2}-\frac{1}{2} u_{2} \Delta u_{1}
$$

where $u_{1}$ and $u_{2}$ denote arbitrary scalar functions. In this way one gets

$$
[\nabla u]^{2}=\frac{1}{2} \Delta\left(u^{2}\right)-u \Delta u
$$

and

$$
\begin{aligned}
\nabla u \cdot \nabla\left([\nabla u]^{2}\right)=\frac{1}{2} \Delta\left(\frac{1}{2} u \Delta\left(u^{2}\right)-u^{2} \Delta u\right)+\frac{1}{2} u \Delta(u \Delta u) & +\frac{1}{2} u(\Delta u)^{2}- \\
& -\frac{1}{4} u \Delta\left(\Delta\left(u^{2}\right)\right)-\frac{1}{4} \Delta u \Delta\left(u^{2}\right) .
\end{aligned}
$$

Taking into account relations (A5) and (A6) the only differential operator appearing in the integrals is the $\Delta$-operator, so that corresponding expressions can be simplified using the eigenvalue relation of spherical harmonics. Subsequently, all integrals needed can be calculated by the use of the orthonormality condition as well as of equations (A1)-(A3). The results of integration are listed below :

$$
\begin{aligned}
& \int u \mathrm{~d} \Omega=\sqrt{4 \pi} U_{00} \\
& \int \Delta u \mathrm{~d} \Omega=0 \\
& \int u^{2} \mathrm{~d} \Omega=\sum_{\ell=0}^{\infty} U_{\ell}^{(2)} \\
& \int u \Delta u \mathrm{~d} \Omega=-\sum_{\ell=0}^{\infty} \ell(\ell+1) U_{\ell}^{(2)} \\
& \int(\Delta u)^{2} \mathrm{~d} \Omega=\sum_{\ell=0}^{\infty} \ell^{2}(\ell+1)^{2} U_{\ell}^{(2)} \\
& \int[\nabla u]^{2} \mathrm{~d} \Omega=\sum_{\ell=0}^{\infty} \ell(\ell+1) U_{\ell}^{(2)} \\
& \int u^{3} \mathrm{~d} \Omega=\sum_{\ell_{1}} \sum_{\ell_{2}} \sum_{\ell_{3}} U \ell_{1}^{(3)}, \ell_{2}, \ell_{3} \\
& \int u^{2} \Delta u \mathrm{~d} \Omega=-\sum_{\ell_{1}} \sum_{\ell_{2}} \sum_{\ell_{3}} \ell_{1}\left(\ell_{1}+1\right) U_{\ell_{1}}^{(3)}, \ell_{2}, \ell_{3} \\
& \int u(\Delta u)^{2} \mathrm{~d} \Omega=\sum_{\ell_{1}} \sum_{\ell_{2}} \sum_{\ell_{3}} \ell_{1}\left(\ell_{1}+1\right) \ell_{2}\left(\ell_{2}+1\right) U_{\ell_{1}}^{(3)}, \ell_{2}, \ell_{3} \\
& \int u[\nabla u]^{2} \mathrm{~d} \Omega=\frac{1}{2} \sum_{\ell_{1}} \sum_{\ell_{2}} \sum_{\ell_{3}} \ell_{1}\left(\ell_{1}+1\right) U_{\ell_{1}, \ell_{2}, \ell_{3}}^{(3)} \\
& \int \nabla u \cdot \nabla\left([\nabla u]^{2}\right) \mathrm{d} \Omega=\sum_{\ell_{1}} \sum_{\ell_{2}} \sum_{\ell_{3}} \ell_{1}\left(\ell_{1}+1\right)\left(-\frac{1}{2} \ell_{1}\left(\ell_{1}+1\right)+\ell_{2}\left(\ell_{2}+1\right)\right) U \ell_{1}^{(3)}, \ell_{2}, \ell_{3}
\end{aligned}
$$


$U_{\ell}^{(2)}$ and $U_{\ell_{1}}^{(3)}, \ell_{2}, \ell_{3}$ are defined in equations (24) and (25), respectively. The conditions for the summations are given in equations (26)-(28).

The nine different integrals appearing in equations (29)-(31) may also be calculated by the use of special properties of spherical harmonics. For that the trigonometric expressions $\sin \vartheta \cos \varphi, \sin \vartheta \sin \varphi$ as well as $\cos \vartheta$ are replaced by corresponding combinations of the $\ell=1$ spherical harmonics $\left(Y_{1 m}\right)$. Then the integrals of first order terms read

$$
\begin{aligned}
\int u \sin \vartheta \cos \varphi \mathrm{d} \Omega & =-\sqrt{\frac{2 \pi}{3}}\left(U_{11}+U_{11}^{*}\right) \\
\int u \sin \vartheta \sin \varphi \mathrm{d} \Omega & =-i \sqrt{\frac{2 \pi}{3}}\left(U_{11}-U_{11}^{*}\right) \\
\int u \cos \vartheta \mathrm{d} \Omega & =\sqrt{\frac{4 \pi}{3}} U_{10} .
\end{aligned}
$$

For the integration of higher order terms it is necessary to consider in detail various symmetry relations of the Clebsch-Gordan coefficients as well as the explicit expressions of these coefficients for $\ell_{2}=1$, i.e. of coefficients $C\left(\ell_{1} 1 \ell_{3} ; m_{1} m_{2}\right)$ (cf. [23]). The resulting integrals may be written as follows:

$$
\begin{aligned}
\int u^{2} \sin \vartheta & \cos \varphi \mathrm{d} \Omega= \\
= & -\sum_{\ell=1}^{\infty} \sum_{m=-1+1}^{\ell-1} \sqrt{\frac{(\ell+m)(\ell+m+1)}{(2 \ell-1)(2 \ell+1)}}\left(U_{\ell, m+1}^{*} U_{\ell-1, m}+U_{\ell, m+1} U_{\ell-1, m}^{*}\right)
\end{aligned}
$$

$$
\begin{aligned}
& \int u^{2} \sin \vartheta \sin \varphi \mathrm{d} \Omega= \\
& =i \sum_{\ell=1}^{\infty} \sum_{m=-\ell+1}^{\ell-1} \sqrt{\frac{(\ell+m)(\ell+m+1)}{(2 \ell-1)(2 \ell+1)}}\left(U_{\ell, m+1}^{*} U_{\ell-1, m}-U_{\ell, m+1} U_{\ell-1, m}^{*}\right)
\end{aligned}
$$

$$
\int u^{2} \cos \vartheta \mathrm{d} \Omega=\sum_{\ell=1}^{\infty} \sum_{m=-\ell+1}^{\ell-1} \sqrt{\frac{\ell^{2}-m^{2}}{(2 \ell-1)(2 \ell+1)}}\left(U_{\ell_{m}}^{*} U_{\ell-1, m}+U_{\ell_{m}} U_{\ell}^{*}-1, m\right)
$$

$$
\begin{aligned}
\int u^{3} \sin \vartheta \cos \varphi \mathrm{d} \Omega=-\frac{1}{2} & \sum_{\ell_{1}} \sum_{\ell_{2}} \sum_{\ell_{3}} \sum_{m_{1}} \sum_{m_{2}} B\left(\ell_{1} \ell_{2} \ell_{3} ; m_{1} m_{2}\right) \times \\
& \times\left(U_{\ell_{1} m_{1}} U_{\ell_{2} m_{2}} U_{\ell_{3}, m_{1}+m_{2}+1}^{*}+U_{\ell_{1} m_{1}}^{*} U_{\ell_{2} m_{2}}^{*} U_{\ell_{3}, m_{1}+m_{2}+1}\right)
\end{aligned}
$$

$$
\begin{aligned}
\int u^{3} \sin \vartheta \sin \varphi \mathrm{d} \Omega=\frac{i}{2} \sum_{\ell_{1}} & \sum_{\ell_{2}} \sum_{\ell_{3}} \sum_{m_{1}} \sum_{m_{2}} B\left(\ell_{1} \ell_{2} \mathfrak{\ell}_{3} ; m_{1} m_{2}\right) \times \\
& \times\left(U_{\ell_{1} m_{1}} U_{\ell_{2} m_{2}} U_{\ell_{3}, m_{1}+m_{2}+1}^{*}-U_{\ell_{1} m_{1}}^{*} U_{\ell_{2} m_{2}}^{*} U_{\ell_{3}, m_{1}+m_{2}+1}\right)
\end{aligned}
$$




$$
\begin{aligned}
\int u^{3} \cos \vartheta \mathrm{d} \Omega & =\sum_{\ell_{1}} \sum_{\ell_{2}} \sum_{\ell_{3}} \sum_{m_{1}} \sum_{m_{2}}\left(U_{\ell_{1} m_{1}} U_{\ell_{2} m_{2}} U_{\ell_{3}, m_{1}+m_{2}}^{*}\right) \times \\
& \times\left(\sqrt{\frac{\left(\ell_{3}-m_{1}-m_{2}\right)\left(\ell_{3}+m_{1}+m_{2}\right)}{\left(2 \ell_{3}-1\right)\left(2 \ell_{3}+1\right)}} A\left(\ell_{1} \ell_{2} \ell_{3}-1 ; m_{1} m_{2}\right)+\right. \\
& \left.+\sqrt{\frac{\left(\ell_{3}+1-m_{1}-m_{2}\right)\left(\ell_{3}+1+m_{1}+m_{2}\right)}{\left(2 \ell_{3}+1\right)\left(2 \ell_{3}+3\right)}} A\left(\ell_{1} \ell_{2} \ell_{3}+1 ; m_{1} m_{2}\right)\right) .
\end{aligned}
$$

In equations (A24) and (A25) the notation $B\left(\ell_{1} \ell_{2} \ell_{3} ; m_{1} m_{2}\right)$ has been used for the following expression :

$$
\begin{aligned}
B\left(\ell_{1} \ell_{2} \ell_{3} ; m_{1} m_{2}\right) & =\sqrt{\frac{\left(\ell_{3}+m_{1}+m_{2}\right)\left(\ell_{3}+1+m_{1}+m_{2}\right)}{\left(2 \ell_{3}-1\right)\left(2 \ell_{3}+1\right)}} A\left(\ell_{1} \ell_{2} \ell_{3}-1 ; m_{1} m_{2}\right)- \\
& -\sqrt{\frac{\left(\ell_{3}-m_{1}-m_{2}\right)\left(\ell_{3}+1-m_{1}-m_{2}\right)}{\left(2 \ell_{3}+1\right)\left(2 \ell_{3}+3\right)}} A\left(\ell_{1} \ell_{2} \ell_{3}+1 ; m_{1} m_{2}\right)
\end{aligned}
$$

Coefficients $A\left(\ell_{1} \ell_{2} \ell_{3} ; m_{1} m_{2}\right)$ are defined by equation (A2). Summations in equations (A24) and (A25) have to be performed under the conditions

$$
\left|m_{i}\right| \leqslant \ell_{1}, \quad(i=1,2) ; \quad\left|m_{1}+m_{2}+1\right| \leqslant \ell_{3}
$$

whereas in equation (A26) the relations (27) and (28) have to be fulfilled. Furthermore, in equations (A26) and (A27) the coefficients $A\left(\ell_{1} \ell_{2} \ell ; m_{1} m_{2}\right),\left(\ell=\ell_{3}-1, \ell_{3}+1\right)$, are zero if one of the conditions (26)-(28) is not fulfilled with $\ell$ replacing $\ell_{3}$ in those conditions.

\section{References}

[1] LIPOWSKY R., Nature 349 (1991) 475.

[2] HELFRICH W., Z. Naturforsch. 28c (1973) 693.

[3] Deuling H. J. and Helfrich W., J. Phys. France 37 (1976) 1335.

[4] Deuling H. J. and Helfrich W., Biophys. J. 16 (1976) 861.

[5] Svetina S. and ŽEKŠ B., Biomed. Biochim. Acta 44 (1985) 979.

[6] Svetina S. and Žekš B., Eur. Biophys. J. 17 (1989) 101.

[7] SheETZ M. P. and Singer S. J., Proc. Nat. Acad. Sci. USA 71 (1974) 4457.

[8] Seifert U., Berndl K. and Lipowsky R., Phys. Rev. A 44 (1991) 1182.

[9] Svetrna S., KRALJ-IgliČ V. and ŽeKš B., Proc. X. School Biophysics of Membrane Transport, J. Kuczera and S. Przestalski Eds., II (Wroclaw, 1990) p. 139.

[10] Svetra S. and ŽEKŠ B., J. Theor. Biol. 146 (1990) 115.

[11] Berndi K., Kas J., Lipowsky R., SaCKMANn E. and SeIfert U., Europhys. Lett. 13 (1990) 659.

[12] Farge E. and DevauX P. F., Biophys. J. 61 (1992) 347.

[13] Helfrich W., J. Phys. France 47 (1986) 321.

[14] Faucon J. F., Mitov M. D., Meleard P., Bivas I. and Bothorel P., J. Phys. France 50 (1989) 2389.

[15] Duwe H. P., Käs J. and Sackmann E., J. Phys. France 51 (1990) 945. 
[16] Courant R. and HILbert D., Methoden der Mathematischen Physik (Verlag von J. Springer, Berlin, 1924).

[17] Milner S. T. and Safran S. A., Phys. Rev. A 36 (1987) 4371.

[18] OU-Yang Zhong-Can and Helfrich W., Phys. Rev. A 39 (1989) 5280.

[19] Pleiner H., Phys. Rev. A 42 (1990) 6060.

[20] Seifert U., J. Phys. A : Math. Gen. 24 (1991) L573.

[21] SEIFERT U., Phys. Rev. Lett. 66 (1991) 2404.

[22] SMIRnow W. J., Lehrgang der höheren Mathematik, Teil II (VEB Deutscher Verlag der Wissenschaften, Berlin, 1963).

[23] Rose M. E., Elementary theory of angular momentum (John Wiley \& Sons Inc., New York and Chapman \& Hall Ltd., London, 1957). 